\title{
Le concept de pression temporelle ultime et sa mesure
}

\author{
Andréa Gourmelen * \\ Maître de conférences \\ IUT Montpellier-Sète, Laboratoire MRM (EA 4557)
}

Bertrand Urien

Professeur des Universités

IAE de Bretagne Occidentale, Laboratoire ICI (EA2652)

Marine Le Gall-Ely

Professeur des Universités

IAE de Bretagne Occidentale, Laboratoire ICI (EA2652)

* Université Montpellier, IUT Montpellier-Sète - Département Techniques de Commercialisation, 99 Avenue d'Occitanie, 34296 Montpellier Cedex 5. Email : andrea.gourmelen@univ-montp2.fr 


\title{
Le concept de pression temporelle ultime et sa mesure
}

\section{Résumé :}

Cette recherche définit la pression temporelle ultime (PTU) comme la conscience d'un temps restant à vivre limité par le rapprochement de la mort et les réactions affectives qui l'accompagnent. Il s'agit d'une nouvelle catégorie de pression temporelle se différenciant des pressions temporelles chronique (PTC) ou situationnelle (PTS). Une échelle de mesure en est proposée dont la validité nomologique est testée sur différentes motivations au bénévolat des retraités, permettant notamment d'aller au-delà de l'utilisation du temps disponible de cette population, comme critère primordial de recrutement et fidélisation de bénévoles.

Mots-clés : bénévolat, échelle de mesure, pression temporelle ultime, retraités, vieillissement.

\section{Ultimate time pressure: conceptualization and measurement}

\begin{abstract}
:
This research defines ultimate time pressure (UTP) as the consciousness of a limited time remaining to live (due to proximity of death) and related feelings. UTP is a new type of time pressure, which differs from chronic time pressure (CTP) and situational time pressure (STP). The objective is to construct a measurement scale, the nomological validity of which will then be used to test the impact of UTP on retiree's motivations to volunteer. Quantifying this influence is important because it will enable us to move away from using "free time" as the main criteria in the recruitment and retention of volunteers from this target population.
\end{abstract}

Key-words: volunteerism, measurement scale, ultimate time pressure, retirees, aging. 


\section{INTRODUCTION}

«Les mois qui nous restent à vivre sont peut-être plus importants que toutes les années passées, et nous devons les vivre dignement» (Tolstoï, « Je m'en vais », Lettres II, 1986: 386).

Le passage à la retraite n'est pas systématiquement vécu comme un tournant de la vie (Lalive d'Epinay et Cavalli, 2007), mais reste considéré comme un élément déclencheur de la prise de conscience du vieillissement (Attias-Donfut, 2006 ; Guiot, 2006). L'individu serait ainsi amené progressivement à réfléchir à sa propre finitude (Szmigin et Carrigan, 2001 ; Urien, 2007), voire à raisonner en termes de durée inversée, c'est-à-dire à se situer dans le temps par rapport à sa mort, et non plus par rapport à sa naissance (Caradec, 2008).

Certaines recherches en marketing s'intéressent à l'impact du rapport à sa propre mort sur les comportements de consommation. Quelques-unes sont centrées sur les aspects cognitifs liés au rapprochement de la mort (Malas et Guiot, 2008 ; Malas et Safraou, 2008), mais abordent peu le ressenti de l'individu. D'autres recherches traitent des affects liés à l'idée de mort (Urien, 2002, 2003), mais ne s'intéressent pas à la proximité perçue de la mort. Ces constats soulèvent des interrogations sur le rapport au temps restant à vivre chez les personnes âgées retraitées, qui doivent faire face à un temps contraint par le rapprochement de leur propre fin. Dans quelle mesure ont-elles conscience du rapprochement de l'échéance ultime ? Eprouventelles uniquement des réactions affectives négatives face à ce rapprochement ? Ces aspects cognitifs et affectifs ont-ils un impact différent sur leurs comportements ? L'objectif de cet article est de proposer le concept de «pression temporelle ultime » (PTU) intégrant à la fois des aspects cognitifs et affectifs liés au temps limité restant à vivre.

Dans une première partie, une revue de littérature sur le vieillissement et le concept générique de pression temporelle permet d'aboutir au concept de pression temporelle ultime. Par la suite, une étude qualitative (partie 2) permet d'approfondir le dimensionnement de la PTU, pour aboutir à une échelle de mesure (partie 3). Enfin, la dernière partie sera consacrée à la validité nomologique et à la portée managériale de ce concept, à travers l'analyse de l'impact de la PTU sur les motivations au bénévolat des retraités. Dans cette recherche, le terme «bénévolat» désigne le bénévolat de type «formel» au sein d'une structure (Erlinghagen, 2010), généralement associative (Prouteau et Wolff, 2004). 


\section{LA PRESSION TEMPORELLE ULTIME : FONDEMENTS THEORIQUES ET CONCEPTUALISATION}

Le rapport au temps restant à vivre et le rapport à la mort chez les individus âgés ont été étudiés, par le biais du vieillissement, en psychanalyse et psychologie clinique, avant de faire leur apparition en marketing. Ces disciplines sources mettent en avant des événements génériques qui jalonnent le vieillissement, notamment la retraite, aboutissant à une prise de conscience accrue du rapprochement de la mort. (1) La psychologie différentielle, centrée sur les différences inter-individuelles et leur mesure (Huteau, 2006), permet d'étudier la diversité des mécanismes de défense permettant de faire face à cette diminution du temps restant à vivre liée au vieillissement. (2) Si la littérature met l'accent sur les aspects cognitifs du rapport au temps restant à vivre, l'existence de mécanismes de défense amène à penser que le rapprochement de la mort ne laisse pas les retraités indifférents émotionnellement. D'où l'intérêt de s'intéresser aux réactions affectives associées à la prise de conscience de ce rapprochement. (3) Le retraité, tiraillé entre regrets liés au temps perdu, peur de manquer de temps avant de mourir et envie de profiter du temps qui lui reste, semble ainsi expérimenter une véritable pression liée au temps restant à vivre : la pression temporelle ultime.

\section{Le processus de vieillissement : origines psychanalytiques et psychologiques}

Initialement, l'approche freudienne ne considère pas le vieillissement comme un phénomène pouvant provoquer des réponses comportementales spécifiques chez les personnes âgées. En effet, cette approche s'attache généralement à relier le comportement à la phase infantile (Gagey, 1989). Cependant, malgré l'importance de la petite enfance dans l'organisation du psychisme, l'individu pourrait évoluer avec le vieillissement. Il serait ainsi affecté par «l'enjeu intime de l'affrontement personnel au temps » (Bianchi, 1989: 2) qui découle du vieillissement. D'ailleurs, en psychologie du développement, les auteurs considèrent que le développement de la personnalité se déroule tout au long de la vie. Selon Erikson (1959), à chaque étape de vie, l'enjeu consiste à trouver un équilibre entre deux pôles extrêmes (positif et négatif). Par exemple, concernant la fin de vie, l'objectif est de s'inscrire sereinement dans le cycle des générations. Pour cela, l'individu doit résoudre un conflit interne entre deux pôles extrêmes : la générativité et la stagnation. Le pôle positif, la générativité, résulte des désirs d'immortalité symbolique et d'être utile aux autres. Ainsi, la générativité est à la fois un geste narcissique, de création de quelque chose à son image, et une action altruiste comme don à la génération suivante (McAdams et al., 1993). Par opposition, le pôle négatif, nommé 
stagnation, représente une «attitude léthargique envers le monde » (Van Hiel et al., 2006: 545). En d'autres termes, cette étape de vie oppose la capacité d'un individu à préserver et guider les générations futures (générativité), à l'impression de n'avoir rien à apporter à ces nouvelles générations (stagnation). Suite à la résolution de ce conflit interne, un nouveau conflit s'opère, cette fois-ci entre « intégrité personnelle » et « désespoir ». L'objectif est alors de réussir à comprendre sa vie pour accepter sa mort. Le vieillissement est ainsi envisagé comme un processus entraînant une succession de bouleversements identitaires. Au cours de cette période, l'individu va se détacher d'objets «narcissisants », c'est-à-dire constitutifs de son identité (Bianchi, 1989). Il va devoir effectuer le deuil de sa vie professionnelle suite à son départ à la retraite (Gagey, 1989; Talpin, 2013), celui de son ancienne image en raison des changements physiques liés à l'âge (Balbo, 1989). Certains auteurs parlent du deuil de la vie (Bianchi, 1989) voire du deuil de soi-même en assumant ses pertes successives (Thomas, 1989). En ce sens, suite au départ à la retraite, le temps libre peut devenir un temps «vide » (Talpin, 2013: 58). Cela signifie un temps inoccupé et donc propice à une introspection, une réflexion sur le sens de sa vie, notamment lorsque l'individu percevait le travail comme un élément indispensable à son bien-être (Marbot et Peretti, 2004). La conscience du rapprochement de la mort, ainsi accrue (Caradec, 2008; Misrahi, 2006), entraînerait des mécanismes de défense conscients et/ou inconscients (Ionescu et al., 2012). Ces derniers sont mis en évidence par les tenants de la théorie de la sélectivité socio-émotionnelle (Carstensen, 1991 ; Reed et Carstensen, 2012), ou de la théorie du management de la terreur (TMT Pyszczynski, Greenberg et Solomon, 1999). Cette théorie précise que tout individu en situation de saillance de mort (confronté à un élément lui rappelant la fragilité de son existence), cherche à se conformer aux valeurs véhiculées par sa culture d'appartenance, entité qui perdurera au-delà de sa propre disparition. Quant à la théorie de la sélectivité socioémotionnelle, elle suggère que le rapprochement de la mort aurait des effets sur le traitement des informations par les personnes âgées. Ces dernières privilégieraient la recherche d'émotions au détriment de la recherche d'informations. De plus, elles auraient tendance à accorder plus d'attention aux informations procurant des émotions positives, à mieux les retenir (par opposition aux messages négatifs). Cependant, pourquoi ce phénomène, appelé « effet de positivité » (Reed et Carstensen, 2012), se produirait-il chez les individus conscients d'un temps restant à vivre limité ? Est-ce parce que la mort est associée à des affects déplaisants (Bianchi, 1987) et que l'individu qui s'en rapproche souhaite diminuer les affects 
négatifs qu'il ressent? Ces interrogations restent en suspens et amènent à décomposer le rapport au temps restant à vivre en éléments d'ordre cognitif et affectif.

\section{Le rapport au temps restant à vivre : des éléments cognitifs et affectifs}

En marketing, Bergadaà (1988, 1989) distingue le temps « ressource» du temps «perspective». Le temps « ressource» correspond à la quantité de temps allouée par un individu à une activité. Le temps «perspective » est subjectif, il fait référence à une perception individuelle (ex: élaboration et planification des buts futurs, préférence pour une zone temporelle, profondeur de l'horizon temporel, etc.). Le rapport au temps restant à vivre, en ce qu'il interpelle l'étendue et le degré d'ouverture de notre horizon temporel, fait partie de ce temps perspective. Existe-t-il des construits relatifs à la perception de ce temps restant à vivre? Sont-ils opérationnalisables?

\section{L'horizon temporel : une variable cognitive traduisant la proximité perçue de la mort} Les prémisses d'une dimension cognitive relative au rapport au temps restant à vivre apparaissent dans les travaux en psychanalyse et en psychologie sur le rapport à la mort chez les personnes âgées. Selon Gagey (1989: 7), la conscience bascule du moriendus (je mourrai certainement un jour) au moriturus (je mourrai bientôt). Le «milieu de la vie » apparaît propice à cette prise de conscience (Bianchi, 1987; Fromage, 2007). Carstensen (1991) approfondit cette idée à travers la théorie de la sélectivité socio-émotionnelle. Celle-ci met en exergue les différences cognitives interindividuelles concernant le temps restant à vivre. Les individus auraient conscience, à des degrés divers, qu'ils se rapprochent de la mort. Ce degré de conscience varierait au fil du temps et impacterait alors les contacts sociaux des personnes âgées. La variable «horizon temporel», censée refléter ce degré de conscience du rapprochement de la mort a été reprise par la suite en marketing (Bourcier-Béquaert et De Barnier, 2011 ; Guiot, 2006). Plus précisément, l’horizon temporel est dit «limité » pour les personnes pensant que leur fin approche (par opposition à l'horizon temporel dit " ouvert» pour les personnes pensant avoir encore beaucoup de temps devant elles). L'aspect cognitif du rapport au temps restant à vivre s'avère alors appréhendé pour partie par l'échelle d'horizon temporel (Carstensen et Lang, 1996). Néanmoins, des problèmes de validité de contenu ont été soulevés concernant cette échelle (Malas et Safraou, 2008). En effet, certains items incluraient des éléments relatifs à l'espoir face à l'avenir ou à des projets dans le futur. Cela va au-delà du concept censé être mesuré. En effet, par définition, l'horizon temporel doit 
mesurer uniquement la proximité perçue de la mort et non la manière dont l'individu espère utiliser son temps restant.

\section{L'existence d'aspects affectifs associés à la proximité perçue de la mort}

Le rapport au temps restant à vivre serait-il uniquement cognitif ? Quelques éléments évoqués dans la littérature permettent d'en douter. Ils ne sont pas mentionnés de manière aussi saillante que les aspects cognitifs, mais plutôt comme des constats, évoqués par certains auteurs, suggérant que le rapprochement de la mort ne laisserait pas les retraités indifférents émotionnellement. Bianchi (1987) indique que la mort est généralement associée à des affects déplaisants. Plus précisément, la littérature en comportement du consommateur s'intéresse aux affects relatifs à l'évocation de sa propre mort. En ce sens, l'anxiété face à la mort est définie comme une gamme de réactions affectives négatives susceptibles de se développer chez un individu confronté à l'idée de sa propre disparition (Urien, 2002, 2003). Si cette littérature ne traite pas de l'horizon temporel considéré, elle met néanmoins en lumière l'existence de réactions affectives négatives apparaissant lorsque sa propre mort est envisagée. Ces considérations amènent à penser que des réactions affectives négatives peuvent être associées aux aspects cognitifs du rapport au temps restant à vivre. Vergely (2010: 108) parle à ce propos «d'épreuve du temps », indiquant que la perception du temps restant à vivre peut faire souffrir, user l'individu. En effet, des regrets ou de la tristesse peuvent apparaître : ce temps restant vaudrait moins la peine d'être vécu que les périodes antérieures durant lesquelles l'individu travaillait, avait moins de soucis de santé etc. (Clément, 2007). Néanmoins, outre la peur de mourir au sens strict du terme, certaines personnes âgées ressentiraient la peur de manquer de temps pour réaliser tout ce qu'elles aimeraient accomplir avant de mourir (De Hennezel, 2010). La conscience de la mort proche susciterait alors la volonté de «mettre à profit sa durée de vie [...] avant que la mort ne lui mette un terme définitif» (Rosa, 2010: 223). En contrepoint de cette littérature, d'autres auteurs mettent en exergue des réactions affectives positives associées à la conscience du rapprochement de la mort et par conséquent du temps restant à vivre limité. Wiedmer (2010) met l'accent sur l'importance accordée à l'envie de profiter de chaque instant, qui deviendrait alors précieux. Ainsi, les personnes âgées retraitées feraient plus attention que les autres à la façon dont elles utilisent ce temps qui leur reste (Szmigin et Carrigan, 2001 ; Warburton et Crosier, 2001). Elles voudraient profiter du reste de la vie ; en gardant à l'esprit qu'elles ne vont pas «durer tout le temps » (Marchand, Quéniart et Charpentier, 2010: 67). Ainsi, la conscience du temps 
restant à vivre limité peut se coupler à l'envie d'en profiter pleinement, de se sentir vivant. Ces éléments affectifs évoqués dans la littérature montrent que certes, la conscience du rapprochement de la mort peut être plus ou moins forte chez deux individus du même âge, mais également que des réactions affectives positives et/ou négatives semblent être associées au degré de conscience du rapprochement de l'échéance ultime.

\section{Intégration des facettes à travers le concept de pression temporelle ultime}

La littérature évoque des éléments cognitifs et affectifs relatifs au rapprochement de l'échéance ultime. Or, à notre connaissance, aucun concept existant n'intègre ces différents aspects. Nous proposons d'introduire un nouveau concept, la pression temporelle ultime (PTU), prenant en compte ces éléments cognitifs et affectifs. Après avoir défini ce nouveau concept, nous le positionnerons par rapport aux autres types de pressions temporelles.

\section{La pression temporelle ultime : un concept cognitivo-affectif}

Bourcier-Béquaert et De Barnier (2012) associent la pression temporelle à la conscience d'un horizon temporel limité chez les personnes âgées. D'après la littérature, l'horizon temporel limité par la mort comme échéance ultime, semble associé à des réactions affectives. Celles-ci seraient positives lorsque l'individu souhaite profiter de chaque instant. Elles seraient négatives, lorsqu'il éprouve des regrets ou craint de ne pas avoir assez de temps avant de mourir. Cela s'apparenterait à une pression temporelle spécifique, liée au temps restant à vivre. En effet, selon la littérature, il existe différentes catégories de pressions temporelles. Cependant, toute pression temporelle se définit comme l'articulation de deux éléments : la conscience d'un temps contraint et les sentiments qui l'accompagnent (Lallement, 2010 ; Szollos, 2009). Ces deux éléments se retrouvent dans le rapport au temps restant à vivre, d'où l'appellation de pression temporelle ultime.

La Pression Temporelle Ultime peut être alors définie comme la prise de conscience d'un temps restant à vivre, limité par le rapprochement de l'échéance ultime (aspect cognitif de la $P T U$ ), et les réactions affectives qui l'accompagnent (aspect affectif de la PTU).

La PTU est donc un type de pression temporelle spécifique. Examinons de plus près sa nature, en la comparant aux autres pressions temporelles.

\section{La pression temporelle ultime, une pression temporelle hybride}

La littérature marketing relative à la pression temporelle est distincte de celle relative au vieillissement. Par conséquent, elle ne traite pas de l'approche de la mort. Deux types de 
pression temporelle sont habituellement distingués (Lallement, 2010 ; Lallement et Machat, 2011) : la «pression temporelle situationnelle » (PTS) et la «pression temporelle chronique » (PTC). La PTS correspond à «l'appréciation individuelle d'avoir ponctuellement un temps insuffisant pour réaliser une tâche » (Lallement, 2010: 48). Elle est donc de courte durée et s'arrête lorsque l'échéance considérée survient (fin d'une promotion commerciale, date d'un événement...)

Par opposition, une «lutte chronique, incessante pour achever de plus en plus de choses en moins en moins de temps » (Rizkalla, 1989: 180) peut perdurer continuellement dans la vie quotidienne de certains individus. Il n'y a pas d'échéance qui provoque cette pression temporelle, qui ne s'arrête ainsi jamais. Progressivement, l'expression «PTC » s'est imposée pour désigner la conscience d'un manque de temps permanent et les sentiments qui l'accompagnent (Lallement et Machat, 2011; Szollos, 2009). Ces derniers sont négatifs (panique, tristesse) lorsque l'individu a l'impression qu'il subit une contrainte temporelle. Inversement, ils sont positifs quand il se sent stimulé par ce manque de temps permanent (Lallement et Machat, 2011 ; Machat et Lallement, 2011).

Par définition, la pression temporelle ultime emprunte des caractéristiques aux autres pressions temporelles : son caractère durable (jusqu'à la mort) la rapproche de la PTC, la présence d'une échéance (en l'occurrence la mort) la rapproche de la PTS. Ni tout à fait chronique, ni tout à fait situationnelle, elle apparaît hybride. Cela permet de la positionner comme une troisième catégorie de pression temporelle en raison de ses caractéristiques distinctives (tableau 1).

\section{< Insérer tableau 1 - Caractéristiques distinctives des pressions temporelles >}

En synthèse, la littérature relative au vieillissement met en exergue l'existence de facettes cognitive et affective relatives au rapport au temps restant à vivre. Il en résulte la nécessité d'aller au-delà du concept d'horizon temporel, ne traitant que d'aspects cognitifs relatifs à l'approche de la mort. Parler de PTU permet d'englober ces deux aspects, car d'une manière générale, tout type de pression temporelle se définit par une combinaison d'aspects cognitifs et affectifs relatifs à un temps contraint. L'approfondissement de la littérature sur la pression temporelle permet alors de considérer la pression temporelle ultime comme une catégorie de pression temporelle à part entière. De ce fait, la PTU est un concept cognitivo-affectif. Cependant, son dimensionnement reste flou. Les réactions affectives sont-elles exclusivement positives ou négatives (l'individu n'éprouverait que l'une ou l'autre des catégories d'affects), 
comme dans le cas de la PTC (Machat et Lallement, 2011) ? Au contraire, une ambivalence psychologique à l'égard du temps restant à vivre peut-elle exister chez les individus (l'individu pourrait éprouver simultanément des affects positifs et négatifs) ? De quels affects s'agit-il ? Une étude qualitative s'avère nécessaire pour clarifier le dimensionnement de la PTU et anticiper la création d'une échelle de mesure.

\section{APPROFONDISSEMENT DU CONCEPT DE PRESSION TEMPORELLE ULTIME, UNE APPROCHE QUALITATIVE}

\section{Méthodologie}

Pour compléter la revue de littérature et délimiter les dimensions de la PTU, une étude qualitative a été réalisée (18 entretiens semi-directifs en face à face, d'une durée moyenne de 55 minutes auprès de retraités âgés de 60 à 86 ans). Les questions relatives au temps restant à vivre ont été insérées au sein d'un guide d'entretien portant également sur le bénévolat. Ce thème permettait à la fois la mise en confiance des répondants et l'approfondissement des relations entre PTU et motivations. Pour éviter tout blocage, des phrases relatives au temps restant à vivre furent présentées aux répondants comme des citations, ou propos d'autres retraités, ce qui s'apparente au test projectif dit de «la troisième personne ». Les entretiens retranscrits ont fait l'objet de résumés, d'une analyse horizontale (Huberman et Miles, 1991) et enfin d'une analyse thématique de contenu (Jolibert et Jourdan, 2011a). Le codage « a priosteriori » a été utilisé. Celui-ci se fonde sur le guide d'entretien mais permet le développement de nouvelles catégories à partir du terrain (Allard-Poesi, 2003). Aussi, l'unité de sens (la plus petite unité permettant de faire sens) a été choisie comme unité de codage (Point et VoynnetFourboul, 2006). Par exemple, lorsqu'un retraité évoquait trois fois une même thématique dans un paragraphe, trois verbatim étaient comptabilisés. Le codage a été effectué via le logiciel Nvivo 10, pour pouvoir utiliser la fonction «fréquence de mots » aidant à la rédaction d'items (Bazeley, 2007). Dans un souci de limitation des biais, l'étude a été évaluée selon les critères de qualité de Miles et Huberman (2003).

\section{Résultats de l'étude qualitative}

L'analyse des entretiens montre que la pression temporelle ultime n'est pas présente de manière identique chez tous les retraités. Des différences individuelles en matière de présence et d'intensité de la PTU ont été observées pour les dimensions cognitive et affective. 
La conscience du rapprochement de l'échéance ultime est exprimée de trois manières différentes (délicate, détournée, décomplexée). Tout d'abord, vraisemblablement en raison du tabou de la mort, des difficultés à aller au-delà de banalités sur le fait que la vie est courte se sont fait ressentir (10 personnes, 16 verbatim). Aussi, certains individus s'expriment sur le sujet de manière détournée, via la mort d'autrui dont ils ont été témoins et qui leur rappelle que l'échéance ultime approche (9 personnes, 16 verbatim) : «On est davantage conscients que... Evidemment on pense à la mort, oui. C'est sûr parce que, il y a des amis qui meurent, avec des tumeurs, tout ça, cancers, qui n'auraient pas dî̀ (Susanne, 65 ans). Certaines personnes s'expriment plus facilement et évoquent leur propre mort de manière décomplexée, spontanément ou après avoir passé par les phases précédentes (9 personnes, 25 verbatim) : " Je pense que j'arrive à la fin de ma vie quand même. Même si je vis encore disons 5, 10 ans, je sais pas »(Jean, 75 ans). "De toute façon c'est simple, une fois qu'on est en retraite, la première chose qu'on se dit, heu, c'est: ah ouais, mais il me reste combien de temps à vivre? »(Joseph, 63 ans). Ce calcul approximatif du temps restant à vivre illustre ainsi le plus fort degré de conscience du rapprochement de la mort, le «moriturus » (je mourrai bientôt) au sens de Gagey (1989: 7).

En outre, les degrés de conscience de la contrainte temporelle ultime apparaissent très variables. En effet, certains répondants se sont exprimés de manière évasive sur ce sujet ou n’ont cessé de relativiser leur temps restant à vivre: «Je pense que j'ai encore des années devant moi logiquement et je peux encore avoir une expérience» (Marie-Danielle, 66 ans). Certaines personnes continuent ainsi d'avoir un horizon temporel ouvert suite à la retraite. Ce résultat, cohérent avec la littérature relative à l'horizon temporel (Malas et Safraou, 2008) montre la pertinence de mesurer ces différences de degrés de conscience du rapprochement de la mort via un instrument approprié.

\section{Les dimensions affectives de la PTU : des sentiments non exclusifs}

Probablement par pudeur et/ou en raison de mécanismes de sélectivité socio-émotionnelle (effet de positivité ${ }^{1}$ - Carstensen, 1991, 1992 ; Reed et Carstensen, 2012), les retraités ont exprimé moins de réactions affectives négatives (68 verbatim) que de réactions affectives positives (128 verbatim) à l'égard de la contrainte de temps restant à vivre.

\footnotetext{
${ }^{1}$ Selon Reed et Carstensen (2012), l'effet de positivité est une particularité du processus cognitif des individus âgés : leur mémoire et leur attention se focalise davantage sur des éléments (informations, images) procurant des réactions affectives positives (par comparaison aux éléments procurant des réactions affectives négatives).
} 
La fin se rapproche, ce qui génère une certaine angoisse en raison de son caractère inconnu. L'individu ne sait pas combien de temps il pourra encore vivre dans les mêmes conditions qu'à l'heure actuelle et faire tout ce qu'il a à faire. "Ce que j'ai peur, c'est que je ne puisse plus aller au jardin quoi. Ça et, là, là, ce serait dur quoi. [...] Comment ça va se passer, je sais pas, ça c'est plus dur » (Mathieu, 74 ans). Ces propos se rapprochent des constats de Clément (2007), relatifs aux regrets liés à la moindre qualité perçue du temps restant à vivre. En effet, ce point brièvement évoqué dans la littérature s'avère bien présent dans les propos des répondants. La majorité des individus concernés s'exprime à travers des comparaisons entre le temps qui reste et le temps vécu. Ils sont tristes d'être déjà arrivés là et expriment de la tristesse, de la mélancolie : "ça nous donne un coup de blues, dire que ah il a déjà cet âgelà, ah oui c'est-à-dire que nous, nous avons ça aussi » (Pierre-Jean, 77 ans). Effectivement, l'individu vit mal le fait de ne plus avoir beaucoup de temps devant lui, il éprouve des regrets et aimerait retourner en arrière : "C'est comme ça, on peut pas revenir en arrière et puis bon, on peut rien faire » (Marie-Danielle, 66 ans). Le temps qui passe apparaît alors subi : «Le temps s'écoule on n'y peut rien on peut pas l'arrêter " (Jean, 75 ans) ; d'où une certaine anxiété liée à l'optimisation du temps restant : «Y a plein d'éléments qui entrent en jeu, mais malgré tout, certains blocages, il faut y aller, il faut y aller, oh non, faut pas attendre » (Joseph, 63 ans).

Ces verbatim complètent ainsi la littérature relativement évasive sur les affects négatifs associés à l'approche de la mort. Si Vergely (2010) évoquait brièvement le fait qu'un individu âgé puisse souffrir à cause du temps restant, l'étude qualitative précise les réactions affectives pouvant conduire à cette souffrance. Ainsi, les affects négatifs relatifs au temps restant à vivre limité mêlent anxiété, crainte de ne pas avoir assez de temps devant soi et regrets, tristesse. En s'apercevant du peu de temps qui lui reste, l'individu regrette la manière dont il a employé son temps par le passé. Il ressent de la tristesse à ce sujet car estime qu'il est déjà trop tard pour certains projets ou activités.

Inversement, comme cela a été suggéré pour la PTC, la contrainte de temps peut avoir un effet stimulant (Lallement et Machat, 2011). Dans le cadre de la PTU, la principale réaction affective positive est l'enthousiasme, le plaisir de savourer ces derniers instants. L'individu apparaît stimulé par la contrainte de l'échéance ultime pour profiter de la vie, comme évoqué dans la littérature (Marchand, Quéniart et Charpentier, 2010) : "Tant qu’on a l'énergie, tant qu'on vit, tant qu'on a envie! Dans envie, il y a vie » (Françoise, 68 ans), pour faire ce dont il 
a envie car il sera bientôt trop tard : "Profitons-en parce que bientôt c'est notre tour de partir » (Pierre-Jean, 77 ans).

La présence de ces éléments d'ordre affectif montre que le rapport au temps restant à vivre s'apparente à une pression temporelle et pas uniquement à un horizon temporel. Effectivement, les éléments cognitifs et affectifs évoqués dans la définition de la PTU s'avèrent présents. De plus, l'étude qualitative présente des résultats cohérents avec la littérature existante sur le vieillissement concernant les réactions affectives positives. Elle précise également les réactions affectives négatives brièvement évoquées par cette littérature. Aussi, bien souvent, les deux types de réactions affectives apparaissent dans les propos d'une même personne, dans des proportions variables. Elles semblent ainsi non-exclusives (13 personnes sur 18 concernées par les deux volets de réactions affectives) à l'inverse de la littérature concernant la PTC (Machat et Lallement, 2011). Nous répondons ainsi à l'interrogation qui subsistait à la fin de la revue de littérature : une ambivalence psychologique semble exister chez les individus âgés concernant les réactions affectives associées à l'approche de la mort.

Etant donné que les deux types de réactions affectives peuvent être présentes chez un même individu, l'éventualité de deux composantes affectives semble alors la plus probable. Cela permet également de prendre en compte la possibilité qu'un individu ne soit pas affecté par la diminution de son temps restant à vivre (un faible score sur les deux composantes affectives refléterait une certaine neutralité). Il en résulte une définition complète du construit PTU : $L a$ pression temporelle ultime est une disposition psychologique qui se manifeste par une dimension cognitive (conscience d'un temps restant à vivre limité) et deux dimensions affectives (négative et positive).

Une étude quantitative s'avère alors nécessaire pour vérifier la structure factorielle de la PTU telle que décrite ci-dessus.

\section{CREATION D’UNE ECHELLE DE MESURE DE LA PRESSION TEMPORELLE ULTIME}

La revue de littérature a permis de mettre en exergue l'existence de facettes cognitive et affective du rapport au temps restant à vivre ; ce qui amène à considérer ce dernier comme une pression temporelle spécifique : la PTU. Or, à notre connaissance, seul le volet cognitif de la PTU a fait l'objet d'une échelle de mesure (horizon temporel), considérée de surcroît comme 
non-opérationnelle (Malas et Safraou, 2008). Pour pouvoir mesurer le rapport au temps restant à vivre sous ses différentes facettes et tester son influence sur les comportements des personnes âgées, il convient donc d'élaborer un instrument de mesure de la pression temporelle ultime (PTU). Pour ce faire, le paradigme de Churchill (1979) et les recommandations de Rossiter $(2002,2011)$ ont été mobilisés.

\section{Spécification du domaine du construit et élaboration des items}

La PTU a été définie comme la prise de conscience d'un temps restant à vivre, limité par le rapprochement de l'échéance ultime (aspect cognitif de la PTU), et les réactions affectives (sentiments) qui l'accompagnent (aspect affectif de la PTU). L'étude qualitative confirme et affine l'existence de ces facettes ; ce qui permet d'envisager une structure tridimensionnelle au regard de l'absence d'exclusivité des réactions affectives (les deux types de réactions affectives peuvent être présentes chez un même individu). En ce sens, une liste de 66 items réflexifs a été élaborée en tenant compte des trois dimensions observées et des verbatim associés à ces dimensions (Rossiter, 2011).

La dimension cognitive représente la conscience d'un temps à vivre contraint par l'échéance ultime. Au sein des verbatim représentatifs de la dimension, prédominent des mots liés à la pensée : le temps passe, la personne y pense, en a conscience. Aussi, figurent des verbes traduisant le rapprochement de l'échéance (approche, arrive), des termes liés à la mort (mort, décès), et au vieillissement (vieux, vieillir). Cette dimension fait ainsi référence à la facette cognitive du rapport au temps restant à vivre évoquée dans la littérature sur l'horizon temporel et permet de répondre à la question «Où suis-je dans le temps (entre ma naissance et ma mort) ? » telle qu'évoquée par Malas et Guiot (2008: 8). En ce sens, les items rédigés reflètent l'horizon temporel de l'individu, afin de mesurer son degré de conscience du rapprochement de la mort.

La dimension affective positive se matérialise par des affects positifs (enthousiasme, stimulation) à l'égard du temps qu'il reste. L'individu perçoit le temps contraint comme une opportunité à saisir, ce qui s'observe à travers des mots tels que : «profiter » ou «envie ». Les adjectifs caractérisant l'émerveillement (beau, superbe) montrent que pour la personne, il est temps de profiter de ce que le monde peut offrir, avant que l'échéance ultime le rattrape. Ces éléments communs à la littérature et à l'étude qualitative nous ont amenés à rédiger des items mettant en parallèle temps restant et stimulation à le savourer de diverses manières. 
La dimension affective négative se matérialise par des affects négatifs (tristesse, craintes) à l'égard du temps qui est compté. Au sein des verbatim classés dans cette dimension, les verbes conjugués au passé montrent la tendance de l'individu à comparer les périodes ; ce qui rapproche cette dimension du concept de regret évoqué par Clément (2007). Le regret est défini dans la littérature comme lié à une comparaison entre la réalité et ce qu'elle aurait pu être, à l'image d'un raisonnement de type «si...alors» (Delacroix et Jourdan, 2007). Si le retraité avait su que le temps passait si vite, par exemple, alors, il aurait géré son temps différemment lors de sa jeunesse et ressentirait moins le besoin d'en gagner aujourd'hui. Craignant un manque qui va se faire davantage sentir à l'avenir, il vit mal sa position sur le spectre temporel et aimerait revenir en arrière pour avoir plus de temps devant lui. Il a néanmoins conscience que ce n'est pas possible, ce qui fait allusion à la notion de «coût irrécupérable», associée d'une manière générale, à un regret (Zeelenberg, 1999: 328). Ces constats amènent à rédiger des items confrontant temps restant et temps passé.

Suite à l'évaluation du degré de représentativité des items par cinq chercheurs confirmés (spécialistes des personnes âgées ou maîtrisant la création d'échelles de mesure) (Jolibert et Jourdan, 2011b) et un prétest auprès de retraités, 39 items se répartissent équitablement entre les dimensions. Il s'agit de ceux possédant la plus grande validité de contenu (Annexe 1).

\section{Epuration et validation de l'échelle de mesure}

\section{Méthodologie des collectes de données}

Deux collectes de données (avec prétest préalable des questionnaires) ont été réalisées (annexe 2: caractéristiques socio-démographiques). La première collecte (janvier $2013 ; 215$ questionnaires) avait pour objectifs d'épurer l'échelle de mesure de la PTU et de tester les instruments de mesure adaptés. La seconde (avril 2013) avait pour objectif de confirmer les structures factorielles (sur une partie de l'échantillon : 180 individus) puis de tester la fiabilité et validité de l'échelle (sur le reste de l'échantillon : 333 individus). Le mode auto-administré a été privilégié au regard du caractère personnel des réponses. Les répondants, recrutés in situ (locaux associatifs, universités du temps libre) ou en ligne (forums, emails à des associations) devaient indiquer leur degré d'accord avec les items. Internet étant de plus en plus familier pour les personnes âgées (Boulbry et al., 2012 ; Chevalier et al., 2013), les répondants pouvaient répondre sur papier ou en ligne.

\section{Analyses factorielles exploratoires et confirmatoires}


Les ACP ont été effectuées sur les échantillons $\mathrm{n}^{\circ} 1(\mathrm{~N}=211, \mathrm{KMO}=0.86)$ et $\mathrm{n}^{\circ} 2(\mathrm{~N}=180$, $\mathrm{KMO}=0.82)$ à l'aide du logiciel SPSS 20. Les items étant censés refléter les dimensions d'un même concept (la PTU), la rotation Oblimin a été choisie (Hair et al., 2010). Sur les deux échantillons, la structure en trois axes semble la plus plausible. Après épurations successives, la structure factorielle se dessine (échelle finale : 11 items, 63\% de variance expliquée), puis est confirmée par AFC, à l'aide du logiciel EQS 6.1. Les trois dimensions : cognitive, affective positive et affective négative apparaissent ainsi stables. La structure factorielle évoquée dans la définition complète de la PTU est donc validée.

Les fortes corrélations entre les dimensions incitent à tester la possibilité d'un modèle de second ordre. Une analyse exploratoire fait apparaître un seul facteur d'ordre supérieur. Ainsi, il est possible de mobiliser le construit latent de second ordre (la PTU au sens global du terme), tout comme de considérer ses trois dimensions, situées à un moindre niveau d'abstraction. Les AFC de premier et second ordre présentent des indices d'ajustement relativement équivalents et supérieurs à ceux de modèles alternatifs (tableau 2). De plus, les loadings standardisés sont significatifs et supérieurs au seuil de 0,5 (annexe 3: structure factorielle).

\section{< Insérer tableau 2 - Résultats des analyses factorielles exploratoires et confirmatoires>}

\section{Fiabilité et validité de l'échelle de mesure}

Les qualités psychométriques de l'instrument de mesure sont vérifiées sur l'échantillon $\mathrm{n}^{\circ} 3$. L'échelle s'avère fiable ( $\alpha$ et $\rho$ de Jöreskog > 0.7). Concernant la validité convergente, les loadings et le test $\mathrm{z}$ dépassent les seuils communément admis, tandis que les $\rho_{\mathrm{vc}}$ avoisinent 0.5. Ainsi, à l'image de Delacroix et Jourdan (2007: 36), observant des $\rho_{\mathrm{vc}}$ entre 0.4 et 0.5 pour certaines dimensions de l'échelle de tendance à regretter du consommateur, les résultats peuvent être considérés comme : «satisfaisants dans le cadre d'une première démarche ».

Pour vérifier la validité discriminante de l'échelle de PTU, les échelles d'anxiété face à la mort (Urien, 2002) et de PTC (Machat et Lallement, 2011) ont été mobilisées (annexe 4). Aucun problème n'a été soulevé pour l'échelle d'anxiété face à la mort. Concernant l'échelle de PTC, un item censé refléter la dimension affective sature sur la dimension cognitive de manière opposée aux autres items. Cet item inversé a probablement posé problème aux individus les plus âgés. Les construits à deux items étant déconseillés dans les modèles d'équations structurelles (Hair et al., 2010), seule la dimension cognitive de la PTC est 
conservée. La proximité théorique entre les construits se traduit par de fortes corrélations. Néanmoins, la validité discriminante est avérée (tableau 3).

\section{<Insérer tableau 3 - Fiabilité et validité de l'échelle PTU >}

La partie suivante, portant sur l'influence de la PTU sur les motivations au bénévolat, va permettre de vérifier la validité nomologique de l'échelle.

\section{ETUDE DE L'INFLUENCE DE LA PTU SUR LES MOTIVATIONS AU BENEVOLAT DES RETRAITES}

La validité nomologique concerne la relation entre le concept mesuré (la PTU) et d'autres concepts qui en découlent logiquement (Jolibert et Jourdan, 2011b), soit ici les motivations au bénévolat. Après avoir expliqué le choix de ce champ d'application, un test de validité nomologique sera effectué par le biais d'un modèle d'équations structurelles.

\section{Utilité de la PTU comme variable explicative des motivations au bénévolat}

Le bénévolat peut remplir une fonction psychique importante suite aux bouleversements liés au vieillissement chez les retraités (Talpin, 2013). Vecteur de lien social, il pourrait conduire vers un vieillissement réussi (Fontaine, 2007). Cependant, dans la réalité, une baisse générale de motivation au bénévolat s'observe lors du passage à la retraite (Cerphi, 2012). Cela peut être dû au fait que certaines associations affectent les bénévoles retraités aux tâches qui requièrent le plus de temps (Van Ingen et Dekker, 2011), sans se soucier de leurs motivations, au motif qu'ils ont du temps disponible. Pourtant, la hausse du temps libre chez les retraités n'entraîne en réalité qu'une augmentation négligeable du temps alloué au bénévolat (Mutchler et al., 2003 ; Prouteau et Wolff, 2007). D'ailleurs, certains retraités craignent de s'engager dans une activité dévoreuse de temps (Tang et al., 2009). Ces constats laissent penser que le rapport qu'entretiennent ces individus face au temps qu'il leur reste à vivre peut jouer un rôle dans le développement de motivations au bénévolat. Si la littérature s'intéresse essentiellement à la classification de ces dernières (Chantal et Vallerand, 2000 ; Clary et al., 1996 ; Okun et al., 1998) et non à leurs déterminants, quelques travaux s'intéressent à leur évolution en fonction de l'âge (Haski-Leventhal, 2009 ; Morrow-Howell, 2010 ; Okun et al., 1998). Parmi ces travaux, certains auteurs (Dávila et Díaz-Morales, 2009 ; Okun et Schultz, 2003 ; O’Neill et al., 2011) mobilisent la théorie de la sélectivité socio-émotionnelle (Carstensen, 1991) à titre de justification a posteriori de certains résultats ; notamment ceux 
traitant de la relation entre âge et motivations relationnelles (retirer des émotions positives comme bénéfice de son action). Or, dans les recherches mentionnant cette théorie, les bénévoles ne sont pas interrogés sur leur rapport au temps restant à vivre. Ce pourquoi, Dávila et Díaz-Morales (2009) évoquent dans leurs pistes de recherche l'intérêt de mobiliser des variables mesurant ce rapport au temps restant (tel que l'horizon temporel). Il apparaît alors intéressant de mobiliser la PTU pour expliquer les motivations au bénévolat des retraités, ce qui est testé ci-après.

\section{Influence de la PTU sur les motivations au bénévolat des retraités : test empirique}

Il existe plusieurs conceptions des motivations des bénévoles. A chaque conception et théorie qui la sous-tend, correspond une échelle de mesure (pour un aperçu des différentes approches, voir annexe 5). L'approche de Chantal et Vallerand (2000) et l'échelle de Motivations envers l'Action Bénévole (EMAB) qui en découle constitue un bon compromis entre les différentes conceptions car elle combine le degré d'altruisme/égoïsme et le degré d'autodétermination (origine de la motivation : choix/contrainte). Ainsi, des items adaptés de l'EMAB reflétant deux motivations opposées (autodéterminées égoïstes et non autodéterminées altruistes) ont été intégrés au questionnaire (annexe 4).

- Autodéterminées égoïstes (origine : soi ; destinataire : soi), soit une volonté de pratiquer une activité bénévole pour en retirer quelque chose (bien-être, augmentation de l'estime de soi, relations...). La littérature évoquée précédemment à ce sujet (Dávila et Díaz-Morales, 2009) permet de suggérer un lien positif entre la dimension affective positive de la PTU et ces motivations au bénévolat. En effet, cette dernière stimulerait l'individu à profiter du temps qu'il lui reste en se faisant plaisir à travers des activités qui lui font du bien. L'étude qualitative montre que le bénévolat peut faire partie de ces activités : «je suis très contente de passer mon temps comme ça, parce qu'il y a une bonne entente, parce que c'est festif [...]. Je ne sais pas quel est l'avenir, moi tant qu'il y a de la vie, je continue »(Françoise, 68 ans). Ainsi, le bénévolat peut être envisagé comme une réponse à cette envie de profiter de la vie évoquée par la littérature; qui correspond à la dimension affective positive de la PTU. Il en résulte l'hypothèse $\mathbf{H 1}$ : La dimension affective positive de la PTU influence positivement les motivations autodéterminées égoïstes.

Cependant, selon Tang et al. (2009), les retraités ne souhaitent pas s'engager dans une activité dévoreuse de temps. La littérature sur le vieillissement permet de comprendre cette 
position. En effet, elle indique que l'envie de profiter de la vie est associée au rapprochement de la mort. Ce dernier conduirait à la volonté de ne pas «gaspiller » ce précieux temps restant (Szmigin et Carrigan, 2001 ; Warburton et Crosier, 2001). Le retraité a conscience de ne plus avoir beaucoup de temps devant lui, ce qui le pousse à le garder pour lui : "J'aimerais en avoir, avoir plus de temps disponible pour mon petit moi égoïste »(Marie-Paule, 62 ans). Ainsi, en dépit du caractère ludique du bénévolat, la conscience d'un temps restant à vivre limité peut freiner l'engouement pour cette activité. D'où H2 : La dimension cognitive de la PTU influence négativement les motivations autodéterminées égoïstes.

- Non autodéterminées altruistes (origine : autrui ; destinataire : autrui), soit une obligation sociale / morale de pratiquer une activité bénévole pour aider son prochain. Cette motivation, à l'opposé de la précédente, serait logiquement sous-tendue par la dimension affective négative de la PTU. En effet, dans les derniers stades de développement, certains individus renoncent à eux-mêmes (Erikson, 1959) en se tournant totalement vers les autres (abnégation), notamment en cas de «bilan de vie négatif» (Talpin, 2013: 43). Le retraité affecté négativement par la diminution de son temps restant à vivre, regrette ainsi le temps qu'il a perdu et la manière dont il l'a employé par le passé. Il pratique alors le bénévolat pour accomplir un devoir moral, employant le temps qui lui reste au service des autres, car les éléments narcissisants, constitutifs du soi, sont malmenés avec le vieillissement : «Je vais partager le sort commun des gens qui prennent de l'âge. Je trouve ça attristant, mais c'est comme ça. Dans le cadre du bénévolat, c'est presque une nécessité sociale de participer à ce qui se passe autour de soi et de faire ce qu'on peut pour essayer [...] de participer à ce qui se passe dans la société »(Marie-Paule, 62 ans). Il en résulte H3: La dimension affective négative de la PTU influence positivement les motivations non autodéterminées altruistes.

Ces hypothèses sont par la suite testées par un modèle d'équations structurelles (figure 1).

\section{<Insérer figure 1 - Influence de la PTU sur les motivations au bénévolat des retraités >}

$\mathrm{H} 1$ et $\mathrm{H} 2$ sont validées. De façon rationnelle, la conscience d'un temps restant à vivre limité rendrait réticent à donner ce temps tandis que les affects positifs liés à cette contrainte poussent l'individu à faire du bénévolat pour profiter de ce temps en compagnie d'autres personnes.

Ces constats s'inscrivent dans le cadre théorique de la sélectivité socio-émotionnelle en y apportant un complément d'information : si les individus âgés choisissent le bénévolat pour 
en retirer quelque chose de positif, ce n'est pas la réduction de leur horizon temporel (dimension cognitive de la PTU) qui est à l'origine de ces motivations (car la relation est négative entre les deux construits), mais bel et bien la sensation de stimulation qui l'accompagne (dimension affective positive de la PTU).

L'hypothèse $\mathrm{H} 3$ relative à l'influence de la dimension affective négative sur les motivations non autodéterminées altruistes est également validée. Affecté négativement par le rapprochement de la mort, le retraité fait preuve d'abnégation. Le soi disparaît, au profit des autres, de la communauté, comme pour rendre à la société ce qu'elle lui a donné. Il anticipe ainsi ce «deuil de soi » évoqué par Thomas (1989: 127).

\section{APPORTS, IMPLICATIONS MANAGERIALES, LIMITES ET VOIES DE RECHERCHE}

Les apports, limites et voies de recherche ouvertes par cet article concernent le concept de PTU et dans une moindre mesure son champ d'application (implications managériales).

\section{Apports théoriques}

Cette recherche permet d'enrichir la littérature par des apports s'inscrivant à la fois dans les travaux sur le vieillissement et dans les recherches sur la pression temporelle.

\section{Vieillissement et aspect tridimensionnel du rapport au temps restant à vivre}

A travers l'étude de la littérature existante et les divers recueils de données effectués, cette recherche montre le caractère incomplet de l'horizon temporel. Ce concept se focalise sur des aspects cognitifs liés à la position perçue de l'individu dans le temps entre sa naissance et sa mort, au détriment de ce qu'il est susceptible de ressentir face au rapprochement de l'échéance ultime. Or, l'existence de réactions affectives associées à des éléments cognitifs - condition nécessaire pour évoquer non plus un horizon temporel (limité), mais bien une pression temporelle spécifique (Lallement, 2010 ; Szollos, 2009) - restait à démontrer. L'un des apports théoriques de cet article réside dans la mise en avant et la mesure de ces aspects affectifs, consubstantiels de la PTU. Aussi, certains auteurs ont souligné le manque de critères temporels pour segmenter les personnes âgées (Moschis et Nickell, 2012), ainsi que d'instruments de mesure adéquats. Ainsi, l'un des enjeux de cette recherche était de proposer un instrument de mesure capable de mesurer le concept de pression temporelle ultime préalablement défini. De surcroît, il s'agissait d'éviter les problèmes de validité de contenu décelés par certains auteurs (Malas, 2009 ; Malas et Safraou, 2008), dans l'échelle d'horizon 
temporel de Carstensen et Lang (1996), antérieure aux recommandations de Rossiter (2002, 2011) à ce sujet.

Pour ce faire, une revue de littérature pluridisciplinaire et une étude qualitative complémentaire ont été nécessaires de manière à créer une échelle de mesure captant toutes les facettes du rapport au temps restant à vivre. Plus précisément, les diverses analyses ont convergé vers un construit tridimensionnel (une composante cognitive, une composante affective positive et une composante affective négative). Ce dimensionnement apparaît cohérent avec la littérature tout en levant le voile sur les réactions affectives liées à l'approche de la mort, qui n'étaient abordées que de manière floue par la littérature.

La composante cognitive de la PTU reflète l'aspect cognitif du rapport au temps restant à vivre qui était d'ores et déjà abordé dans la littérature sur le vieillissement à travers le concept d'horizon temporel. Les quatre items abordent ainsi la notion de conscience de la proximité de la mort, de son rapprochement suite à l'avancée en âge (Caradec, 2008 ; Gagey, 1989). En outre, l'étude qualitative a permis de confirmer l'existence de différences individuelles de degrés de conscience du rapprochement de l'échéance ultime, évoquées par la théorie de la sélectivité socio-émotionnelle (Carstensen, 1991). Les items permettent ainsi de mesurer jusqu'à quel point un individu âgé a conscience qu'il se rapproche de la mort, c'est-à-dire la profondeur de son horizon temporel et uniquement cela, palliant ainsi les limites de l'échelle de Carstensen et Lang (1996) soulignées par Malas et Safraou (2008).

Les deux autres composantes sont d'ordre affectif et complètent ainsi l'horizon temporel qui n'aborde pas les ressentis individuels face à la proximité perçue de la mort.

La composante affective positive reflète ce regain de vie, associé à la proximité de la mort, évoqué dans la littérature sur le vieillissement (Marchand, Quéniart et Charpentier, 2010 ; Wiedmer, 2010). L'étude qualitative a permis de préciser la nature de cette composante affective : l'envie de profiter de la vie y apparaît centrale, comme si l'individu était stimulé par la dernière opportunité du temps restant à vivre pour accomplir tout ce qui lui est encore possible. Le désir de vivre pleinement ces derniers instants se traduit alors dans les items de cette dimension. La mise en parallèle de cette stimulation et du fait de penser au temps qui passe permet de parler d'une pression temporelle. En effet, ces affects positifs accompagnent bien une pensée relative à un temps contraint, comme pour toute pression temporelle: l'individu souhaite profiter pleinement du temps restant car celui-ci est compté. Si la littérature semblait d'ores et déjà souligner cette forte imbrication entre cognition et affects 
positifs liés au temps restant à vivre (ex : Marchand, Quéniart et Charpentier, 2010: 67 évoquent le fait que les personnes âgées souhaitent profiter de la vie en gardant à l'esprit qu'elles ne vont pas «durer tout le temps»), aucun instrument de mesure ne permettait d'appréhender ces affects positifs jusqu'alors.

La composante affective négative était quant à elle, la plus floue dans la littérature. Certains auteurs soulignent en effet l'existence de réactions affectives négatives associées à l'idée de la mort en général (Bianchi, 1987), de sa propre mort (Urien, 2002, 2003), mais qu'en est-il de celles associées à la proximité perçue de sa propre mort ? La littérature évoque succinctement une gamme de réactions affectives négatives associées au rapprochement de l'échéance ultime, telles que la peur de manquer de temps avant de mourir (De Hennezel, 2010), la tristesse, les regrets (Clément, 2007). Le temps restant à vivre ferait ainsi souffrir (Vergely, 2010). L'étude qualitative a permis de clarifier la place de ces réactions affectives négatives entremêlées. Les regrets semblent ainsi liés au temps perdu lorsque l'individu était plus jeune, à l'impression d'avoir gaspillé du temps, à l'époque où ce dernier n'était pas encore « compté ». L'individu serait alors à la fois triste et anxieux à l'idée de se représenter le temps restant : triste par rapport à sa position temporelle et l'impossibilité de revenir en arrière ; anxieux par rapport à ce qu'il pourra accomplir dans ce temps limité avant de mourir. Les items de la dimension reflètent ainsi ces différentes réactions affectives associées au temps restant à vivre, par opposition à celui vécu.

\section{La pression temporelle ultime comme troisième catégorie de pression temporelle}

Concernant le second volet de la littérature : la pression temporelle, cette recherche permet d'enrichir les catégories existantes. En effet, la pression temporelle est une variable qui a été clarifiée relativement récemment (Lallement, 2008, 2010 ; Lallement et Machat, 2011 ; Machat et Lallement, 2011). Cette littérature ne traitant pas des ressentis possibles associés à l'approche de la mort, les recherches se sont limitées à explorer des conditions de temps contraint dans la vie des individus. Ainsi, deux grandes catégories étaient généralement distinguées jusqu'alors :

- la PTS (Lallement, 2008, 2010), soit la pression temporelle de courte durée expérimentée par un individu devant faire face à une échéance dans un futur relativement proche (ex : date d'anniversaire pour effectuer un achat, fin d'une promotion commerciale) 
- la PTC (Lallement et Machat, 2011 ; Machat et Lallement, 2011 ; Szollos, 2009), soit la pression temporelle de longue durée, reflétant un manque de temps constaté au quotidien et les réactions affectives qui l'accompagnent (stimulation ou frustration).

Cependant, la mort peut être considérée comme une échéance (l'échéance ultime) et ainsi faire l'objet d'une pression temporelle lorsque l'individu perçoit son rapprochement. Ni situationnelle, ni chronique, la pression temporelle ultime apparaît comme complémentaire à ces deux catégories. En effet, la littérature n'a jamais considéré la dichotomie formée des pressions temporelles situationnelle et chronique comme une liste exhaustive des pressions temporelles existantes. Cette recherche permet d'en ajouter une : la pression temporelle ultime ; relative à une échéance particulière (celle de sa propre mort), qui dure du moment de la prise de conscience de son rapprochement au décès de l'individu concerné.

\section{Implications managériales}

Le bénévolat constitue un premier champ d'application pour la PTU. Il en résulte des implications pour les associations, cherchant à fidéliser les bénévoles retraités pour lutter contre le turn-over (Cerphi, 2010). En effet, cette recherche montre un lien entre la dimension positive de la PTU et les motivations autodéterminées égoïstes (relations, découverte, bienêtre etc.). Il conviendrait ainsi, dans la communication, d'intégrer les aspects positifs du temps restant à vivre à travers des références à une «nouvelle vie», « un temps pour s'épanouir ». Ces éléments ne sont pas sans rappeler "l'identity renaissance» de Schau, Gilly et Wolfinbarger (2009), c'est-à-dire la renaissance d'une identité de consommateur suite à la disparition de l'identité professionnelle chez les retraités. La consommation d'expériences de bénévolat permettrait ainsi un renouveau, un ajout d'éléments à son histoire de vie. Ces allusions à la dimension affective de la PTU, associées à des missions telles que l'organisation de manifestations sportives ou culturelles, pourraient alors attirer ces retraités en quête d'expériences enrichissantes sur le plan psychosocial.

Inversement, la PTU affective négative conduit à un bénévolat animé par l'obligation morale d'aider son prochain. Ainsi, présenter la retraite comme un temps utile au service des autres semble intéressant pour promouvoir des actions de type "collecte de denrées alimentaires » ou de vêtements, sous-tendues par le devoir de rendre à la société ce qu'elle nous a donné.

Le test de validité nomologique s'étant avéré concluant, un modèle plus complet permettrait d'intégrer un plus large spectre de motivations (Chantal et Vallerand, 2000) et d'identifier des variables modératrices. Les états dépressifs associés à des événements liés au vieillissement 
(Fontaine, 2007), tels que des deuils, des maladies, pourraient exercer un effet modérateur entre PTU et motivations au bénévolat, au sens où ils inhiberaient toute motivation à agir. Quant à l'attitude d'acteur ou de réacteur face au temps (Bergadaà, 1990 ; Bergadaà et Urien, 2008 - sur le don d'objets), qui mesure la manière dont les individus anticipent leur futur, elle pourrait avoir un rôle modérateur entre les motivations et le comportement de bénévolat.

Enfin, la générativité, mobilisée dans les travaux antérieurs sur le bénévolat des retraités (Gourmelen et Guillemot, 2012), pourrait être envisagée en complément de la PTU. Cela permettrait de considérer davantage le bénévolat comme une réponse comportementale aux enjeux développementaux des derniers stades d'Erikson (1959).

\section{Limites et voies de recherche futures}

\section{Elargissement à d'autres populations}

L'échelle de mesure de la PTU a été construite en mobilisant un échantillon de retraités français. Or, malgré l'universalité supposée de la théorie de la sélectivité socio-émotionnelle de Carstensen (Malas et Safraou, 2008), la façon de se représenter le temps est impactée par le contexte culturel. Dans la culture occidentale, le temps se représente comme une droite, une flèche, alors que dans la culture orientale, la représentation du temps s'apparenterait à un cercle, un cycle, à l'image de celui des saisons (Adjaye, 2002 ; Usunier et Valette-Florence, 2007). Or, la PTU est associée à la position perçue d'un individu sur la flèche du temps. La PTU est-elle similaire chez les individus ayant une perception cyclique du temps ?

De plus, dès lors que l'échelle possède une bonne fiabilité et validité, il serait intéressant d'étudier l'intensité de la PTU chez des individus de diverses tranches d'âge. En effet, dans le cas de personnes relativement jeunes ayant vécu des événements caractéristiques du vieillissement (ex : décès de parents ou d'amis de la même génération), la PTU est-elle présente? Des tests projectifs plus élaborés pourraient alors s'avérer nécessaires en complément de l'administration de questionnaires en face à face, afin de vérifier si les mécanismes de défense ne sont pas trop importants chez ces individus. En effet, les individus âgés s'expriment plus facilement au sujet de la mort que des individus plus jeunes (l'anxiété face à la mort étant plus élevée aux alentours de la quarantaine - Urien, 2003).

\section{Antécédents de la PTU}

Dans la lignée du constat précédent concernant la possibilité de PTU chez des individus plus jeunes, les résultats obtenus lors du test de validité discriminante incitent à ancrer la PTU dans un réseau nomologique plus complet. Si des variables ont été évoquées précédemment comme 
pistes de recherche futures sur le bénévolat, d'autres concepts pourraient être étudiés indépendamment du champ d'application. En effet, cette recherche montre que les retraités n'appréhendent pas l'échéance ultime de manière identique. Comment expliquer ces différences ? Les fortes corrélations entre PTU, anxiété face à la mort et peur du vieillissement incitent à approfondir les recherches sur l'articulation entre ces concepts. L'anxiété face à la mort et la peur du vieillissement peuvent-elles être considérées comme des antécédents de la PTU ? Un individu, qui, avant la retraite, développe une forte anxiété face à la mort, par exemple, aura-t-il une probabilité plus forte qu'un autre de développer des affects négatifs liés au rapprochement de l'échéance ultime? D'autres traits de personnalité stables dans le temps, comme la sensibilité au regret (Schwartz et al., 2002), pourraient expliquer la prédominance de la dimension affective négative de la PTU chez certains individus, car cette dernière fait allusion à des regrets relatifs au temps perdu.

\section{Portée managériale de la PTU}

Les implications managériales de cette recherche concernent principalement le champ d'application étudié, le bénévolat des retraités. Une piste de recherche intéressante serait de développer la portée opérationnelle du construit PTU en lui-même. Pour ce faire, une typologie de retraités basée uniquement sur les trois dimensions de la PTU pourrait conduire à des segments génériques de retraités selon leur rapport au temps restant à vivre. Cette recherche montre effectivement que les trois dimensions de la PTU sont présentes chez les retraités à des degrés divers et peuvent influencer de manière différente leurs comportements. Une typologie permettrait de déterminer s'il existe ou non des profils de retraités marqués par une ou deux dimensions dominantes. Cela faciliterait le ciblage des retraités par toute organisation. Certains individus peuvent-ils avoir une dominante cognitive de la PTU, c'est-àdire être relativement indifférents au rapprochement de la mort? Quant aux réactions affectives, si l'étude qualitative a montré l'existence d'une ambivalence psychologique chez la majorité des répondants, certains retraités peuvent-ils être dominés par le volet positif ou négatif de ces réactions affectives ? L'idée sous-jacente à la création d'une typologie serait de faciliter le ciblage et la communication sur le temps restant à vivre, relativement délicate à mettre en œuvre, en dégageant des profils dominés par différentes dimensions de la PTU et en les qualifiant à l'aide de variables socio-démographiques.

Par ailleurs, il conviendrait de tester l'influence de la PTU sur d'autres champs d'application que le bénévolat afin de développer sa portée managériale. Les choix financiers des retraités, 
notamment l'arbitrage entre consommation et épargne, ont d'ores et déjà été étudiés sous le prisme du temps, à travers la variable « horizon temporel» (Malas et Guiot, 2008 ; Malas et Safraou, 2008; Malas, 2009). Or, au-delà de la proximité perçue de la mort, il serait intéressant d'examiner l'impact des réactions affectives positives et négatives associées sur les décisions financières des personnes âgées. La dimension affective positive reflétant une envie de profiter du temps restant pourrait impacter des décisions d'achat (voyages, loisirs) chez les retraités, au détriment de l'épargne destinée aux générations suivantes, par exemple. Une typologie fondée sur le rapport au temps restant à vivre associée au rapport à l'argent prendrait ainsi tout son sens appliquée à ce champ de recherche. 


\section{Annexe 1 : Items générés retenus par les experts et processus d'épuration}

Le tableau suivant présente les items ayant passé l'étape de la validité de contenu et le stade du processus d'épuration auquel ils ont été éliminés.

La lettre E signifie que l'item a été éliminé suite aux analyses en composantes principales, la lettre $\mathrm{C}$ signifie que l'item a été conservé (l'échantillon n³ n’apparaît pas car aucun item n’a été supprimé lors de l'ACP effectuée sur ce dernier échantillon). Les items en gras sont ceux de la structure factorielle finale.

\section{Libellé item}

Echantillon 1 tillon 2

\section{Dimension cognitive}

A mon âge, il faut s'attendre à mourir dans un avenir proche

J'estime avoir encore beaucoup de temps devant moi (inverse)

A mesure que je vieillis, je commence à m'apercevoir que mon temps est compté

A mon âge, l'avenir se résume à quelques années

Je pense que la fin de ma vie est proche

Je suis encore jeune pour penser à la dépendance (inverse)

Plus j'avance en âge, plus je me dis que la mort se rapproche

J'évite de penser au temps qu'il me reste à vivre (inverse)

Combien d'années vais-je encore vivre ? Est une question que je me pose souvent

Dimension affective positive

Je vieillis, alors tant qu'il me reste l'énergie, j'en profite pour faire ce qu'il me plaît

Peu importe le temps qui passe, je me sens plus épanoui(e) qu'avant

Quand on a la vie devant soi, on court toujours après quelque chose. En vieillissant, on vit plus posément

La santé ne tient qu'à un fil, alors à mon âge, il ne faut pas trop attendre pour réaliser certaines choses

Tant qu'on est vivant, il faut garder la joie de vivre quoi qu'il arrive

Quand on voit s'approcher la fin, cela motive pour profiter de la vie

Le bénéfice de l'âge, c'est de savourer le temps que l'on a pour soi-même jusqu'à la fin

J'aime vivre au jour le jour, car la vie peut s'arrêter du jour au lendemain

Penser au temps qui passe me stimule pour aller au bout de mes envies

Vu le temps qui me reste, j'envisage l'avenir comme une succession de moments de bonheur avec mes proches

Le fait de penser à la fin de la vie me permet de vivre intensément les moments présents

Plus j'avance en âge, plus je me sens libéré des contraintes horaires

A la retraite, c'est le moment de se laisser vivre avant qu'il ne soit trop tard

\begin{tabular}{|l|l|}
\hline $\mathrm{C}$ & $\mathrm{C}$ \\
$\mathrm{C}$ & $\mathrm{E}$ \\
\hline $\mathrm{E}$ & \\
$\mathrm{C}$ & $\mathrm{C}$ \\
\hline $\mathrm{C}$ & $\mathrm{C}$ \\
\hline $\mathrm{E}$ & \\
\hline $\mathrm{C}$ & $\mathrm{C}$ \\
\hline $\mathrm{E}$ & \\
\hline $\mathrm{E}$ & \\
\hline
\end{tabular}

Dimension affective négative

Penser au temps qui passe, c'est désagréable, surtout passé un certain âge

Quand je vois les enfants qui grandissent tellement vite, ça me donne un coup de blues

Le fait de devoir me ménager quand je prendrai de l'âge me fait peur

Ça m'attriste de me dire que mon rythme de vie va se ralentir au fil des années

Penser aux années qu'il me reste me rend mal à l'aise

J'ai du chagrin quand je me rends compte que j'ai déjà cet âge-là

Quand je pense à l'âge que j'aurai dans cinq ans, ça ne me réjouit pas

Je regrette de ne pas avoir été jusqu'au bout de certains projets quand il était encore temps

Si j'avais su que le temps passait si vite, j'aurais profité davantage de ma jeunesse

Je suis effrayé(e) par le fait que la vie passe si vite

J'ai de la peine quand je pense à certaines activités que je ne peux plus faire

J'aimerais rattraper tout le temps que j’ai perdu par le passé, car je crains d'en manquer.

On ne devrait pas vieillir, c'est trop triste

Je crains d'être de moins en moins capable d'être actif avec l'âge

Le temps passe tellement vite, il faudrait malheureusement plusieurs vies pour faire tout ce qu'on veut

Je crains de ne pas pouvoir faire tout ce que j'ai à faire avant de mourir

Je me sens pressé de réaliser certaines choses avant qu'il ne soit trop tard

\begin{tabular}{|c|c|}
\hline $\mathrm{E}$ & \\
$\mathrm{E}$ & \\
$\mathrm{E}$ & \\
$\mathrm{E}$ & \\
$\mathrm{E}$ & \\
\hline $\mathrm{C}$ & $\mathrm{C}$ \\
\hline $\mathrm{E}$ & \\
\hline $\mathrm{C}$ & $\mathrm{E}$ \\
\hline $\mathrm{C}$ & $\mathrm{C}$ \\
\hline $\mathrm{E}$ & \\
\hline $\mathrm{C}$ & $\mathrm{C}$ \\
\hline $\mathrm{C}$ & $\mathrm{E}$ \\
\hline $\mathrm{E}$ & \\
\hline
\end{tabular}

\begin{tabular}{|l|l|}
\hline E & \\
\hline C & E \\
\hline E & \\
\hline E & \\
\hline E & \\
\hline E & \\
\hline E & \\
\hline$C$ & $C$ \\
\hline$C$ & $C$ \\
\hline E & \\
\hline E & \\
\hline$C$ & $C$ \\
\hline$C$ & E \\
\hline E & \\
\hline$C$ & E \\
\hline$C$ & $C$ \\
\hline$C$ & E \\
\hline
\end{tabular}


Annexe 2 : Caractéristiques socio-démographiques des répondants

\begin{tabular}{|c|c|c|c|}
\hline & $\begin{array}{c}\text { Echantillon } 1 \\
\qquad(\mathrm{~N}=\mathbf{2 1 1})\end{array}$ & $\begin{array}{c}\text { Echantillon } 2 \\
(\mathrm{~N}=180)\end{array}$ & $\begin{array}{c}\text { Echantillon } 3 \\
\quad(\mathrm{~N}=333)\end{array}$ \\
\hline \multicolumn{4}{|c|}{ Age } \\
\hline Age moyen & 68 ans & 66,3 ans & 67,2 ans \\
\hline Ecart-type & 6,739 & 4,980 & 5,75 \\
\hline Minimum & 54 ans & 55 ans & 54 ans \\
\hline Maximum & 87 ans & 83 ans & 87 ans \\
\hline \multicolumn{4}{|c|}{ Genre } \\
\hline Hommes & $49,3 \%$ & $52,8 \%$ & $50,2 \%$ \\
\hline Femmes & $50,7 \%$ & $46,7 \%$ & $49,8 \%$ \\
\hline $\mathrm{NC}$ & $0 \%$ & $0,6 \%$ & $0 \%$ \\
\hline \multicolumn{4}{|c|}{ Niveau d'études* } \\
\hline Enseignement primaire & $2 \%$ & $2,2 \%$ & $2 \%$ \\
\hline Brevet, certificat d'études & $13 \%$ & $8,9 \%$ & $8 \%$ \\
\hline BEP, CAP & $22 \%$ & $16,7 \%$ & $19 \%$ \\
\hline BAC et niveau BAC & $18 \%$ & $23,9 \%$ & $18 \%$ \\
\hline $\mathrm{BAC}+1,2$ et 3 & $27 \%$ & $28,3 \%$ & $27 \%$ \\
\hline $\mathrm{BAC}+4$ et plus & $18 \%$ & $19,4 \%$ & $26 \%$ \\
\hline $\mathrm{NC}$ & $0 \%$ & $0,6 \%$ & $0 \%$ \\
\hline \multicolumn{4}{|c|}{ Statut de retraité*** } \\
\hline Oui & $100 \%$ & $100 \%$ & $100 \%$ \\
\hline Non & $0 \%$ & $0 \%$ & $0 \%$ \\
\hline
\end{tabular}

* Le niveau d'études apparaît relativement élevé compte tenu de l'âge de la population étudiée. Cela apparaît cohérent avec la littérature antérieure sur le sujet (Erlinghagen et Hank, 2005 ; Zedlewski et Schaner, 2006), qui constate un niveau d'études plus élevé chez les personnes âgées bénévoles.

**Rappelons qu'il n'y avait pas de limite d'âge pour répondre à notre questionnaire. Seul le fait d'être retraité était une condition pour pouvoir participer à la recherche. Ainsi, tous les individus, même ceux ayant moins de 60 ans (minoritaires : 5,8\% de notre échantillon global), sont retraités. 
Annexe 3 : Structure factorielle, échelle PTU, échantillons $n^{\circ} 2$ et 3

\begin{tabular}{|c|c|c|c|c|c|c|}
\hline \multirow[b]{3}{*}{$\begin{array}{l}\text { J'aimerais rattraper tout le temps que j'ai perdu } \\
\text { par le passé, car je crains d'en manquer }\end{array}$} & \multicolumn{6}{|c|}{ Composante } \\
\hline & \multicolumn{2}{|c|}{$\begin{array}{l}1 \text { (affective } \\
\text { négative) }\end{array}$} & \multicolumn{2}{|c|}{2 (cognitive) } & \multicolumn{2}{|c|}{$\begin{array}{l}3 \text { (affective } \\
\text { positive) }\end{array}$} \\
\hline & $0,805^{*}$ & $0,81 * *$ & & & & \\
\hline $\begin{array}{l}\text { Je regrette de ne pas avoir été jusqu'au bout de } \\
\text { certains projets quand il était encore temps }\end{array}$ & 0,777 & 0,62 & & & & \\
\hline $\begin{array}{l}\text { Si j'avais su que le temps passait si vite, } \\
\text { j'aurais profité davantage de ma jeunesse }\end{array}$ & 0,772 & 0,69 & & & & \\
\hline $\begin{array}{l}\text { Je crains de ne pas pouvoir faire tout ce que } \\
\text { j'ai à faire avant de mourir }\end{array}$ & 0,680 & 0,58 & & & & \\
\hline $\begin{array}{l}\text { A mon âge, il faut s'attendre à mourir dans un } \\
\text { avenir proche }\end{array}$ & & & $0,877 *$ & $0,82 * *$ & & \\
\hline Je pense que la fin de ma vie est proche & & & 0,783 & 0,75 & & \\
\hline $\begin{array}{l}\text { A mon âge, l'avenir se résume à quelques } \\
\text { années }\end{array}$ & & & 0,755 & 0,80 & & \\
\hline $\begin{array}{l}\text { Plus j'avance en âge, plus je me dis que la } \\
\text { mort se rapproche }\end{array}$ & & & 0,684 & 0,62 & & \\
\hline $\begin{array}{l}\text { Quand on voit s'approcher la fin, cela motive } \\
\text { pour profiter de la vie }\end{array}$ & & & & & $0,849 *$ & $0,78 * *$ \\
\hline $\begin{array}{l}\text { Le fait de penser à la fin de la vie me permet } \\
\text { de vivre intensément les moments présents }\end{array}$ & & & & & 0,791 & 0,77 \\
\hline $\begin{array}{l}\text { Penser au temps qui passe me stimule pour } \\
\text { aller au bout de mes envies }\end{array}$ & & & & & 0,784 & 0,75 \\
\hline
\end{tabular}

* Loadings matrice des types ACP échantillon $\mathrm{n}^{\circ} 2$

** Loadings standardisés AFC de $2^{\text {nd }}$ ordre échantillon $n^{\circ} 3$. 


\section{Annexe 4 : La mesure des autres construits}

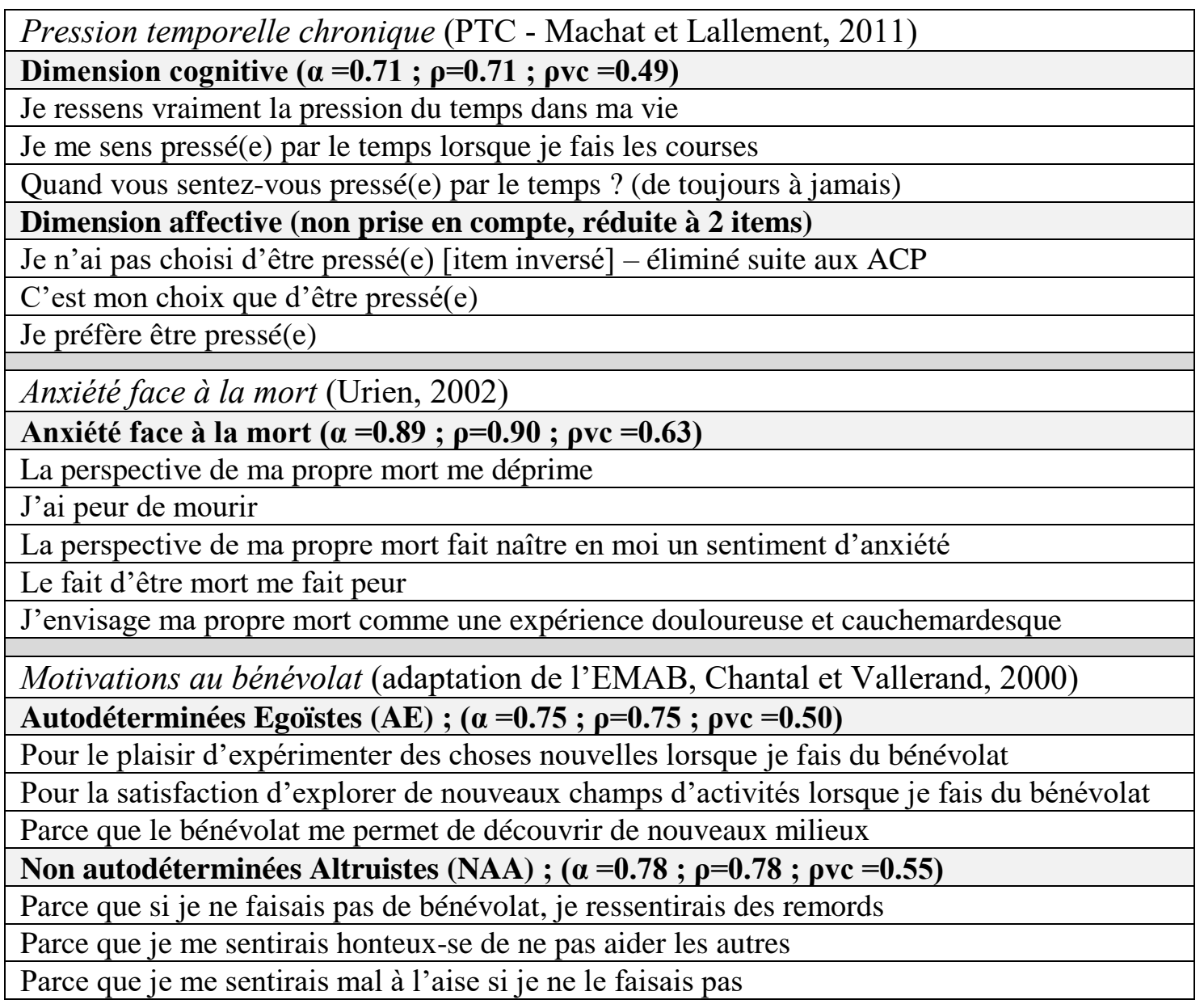




\section{Annexe 5 : Mesure des motivations au bénévolat, choix de l'approche de Chantal et Vallerand (2000)}

\begin{tabular}{|c|c|c|c|c|}
\hline Echelle & $\begin{array}{l}\text { Volunteer } \quad \text { Functions } \\
\text { Inventory (VFI, Clary et al., } \\
\text { 1996) }\end{array}$ & $\begin{array}{l}\begin{array}{l}\text { Echelle } \\
\text { motivations }\end{array} \begin{array}{r}\text { de } \\
\text { envers } \\
\text { l'action bénévole }\end{array} \\
\text { (EMAB, Chantal et } \\
\text { Vallerand, 2000) } \\
\end{array}$ & $\begin{array}{l}\text { Motivations for sports } \\
\text { volunteerism } \\
\text { (Wang, }\end{array}$ & $\begin{array}{l}\text { Motivation at work scale } \\
\text { revised (MAWS-R) Gagné et } \\
\text { al., } 2015\end{array}$ \\
\hline Discipline & Psychologie & Psychologie & Marketing & Psychologie \\
\hline $\begin{array}{l}\text { Fondements } \\
\text { théoriques }\end{array}$ & $\begin{array}{l}\text { Théorie fonctionnaliste (un } \\
\text { bénévole peut poursuivre } \\
\text { plusieurs buts et deux } \\
\text { personnes peuvent s'engager } \\
\text { dans la même activité sans } \\
\text { pour autant avoir les mêmes } \\
\text { motivations) }\end{array}$ & $\begin{array}{l}\text { Théorie de } \\
\text { l'autodétermination } \\
\text { (faire du bénévolat par } \\
\text { choix ou contrainte) + } \\
\text { Dichotomie altruisme / } \\
\text { égoïsme des } \\
\text { motivations }\end{array}$ & Théorie fonctionnaliste & $\begin{array}{l}\text { Théorie } \\
\text { l'autodétermination }\end{array}$ \\
\hline $\begin{array}{l}\text { Points } \\
\text { positifs }\end{array}$ & $\begin{array}{l}\text { Popularité (nombreux tests), } \\
\text { simplicité. }\end{array}$ & $\begin{array}{l}\text { Tient compte à la fois } \\
\text { de l'origine de la } \\
\text { motivation et du } \\
\text { destinataire de l'action. }\end{array}$ & $\begin{array}{lr}\text { Utilisation de } & \text { théories } \\
\text { psychologiques } & \text { en } \\
\text { marketing } & \end{array}$ & $\begin{array}{l}\text { Montre l'existence d'un regain } \\
\text { d'intérêt pour la théorie de } \\
\text { l'autodétermination dans la } \\
\text { littérature. }\end{array}$ \\
\hline Limites & $\begin{array}{l}\text { Certains auteurs regroupent } \\
\text { des dimensions de l'échelle } \\
\text { selon des critères liés au degré } \\
\text { d'altruisme / égoïsme } \\
\text { (Agostinho et Paço, 2012) ou } \\
\text { au degré d'autodétermination } \\
\text { (Finkelstein, 2009). }\end{array}$ & 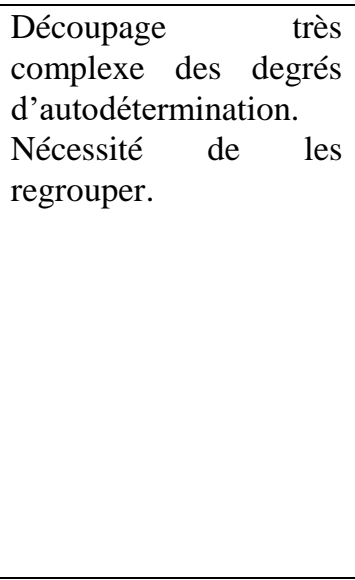 & $\begin{array}{l}\text { Trop spécifique au } \\
\text { bénévolat sportif } \\
\text { événementiel. } \\
\text { Mélange d'items traitant } \\
\text { de motivations actuelles } \\
\text { (ex : «Je fais du } \\
\text { bénévolat parce que } \\
\text { j'aime aider les autres ») } \\
\text {; futures (ex : « } \\
\text { j'aimerais faire partie de } \\
\text { l'événement ») et } \\
\text { d'opinions (ex: "le } \\
\text { bénévolat est un service } \\
\text { rendu à la communauté » }\end{array}$ & $\begin{array}{l}\text { Echelle utilisée par certains } \\
\text { auteurs (Haivas, Hofmans et } \\
\text { Pepermans, 2012; Bidee et } \\
\text { al., 2013) pour mesurer les } \\
\text { motivations au bénévolat mais } \\
\text { initialement conçue pour } \\
\text { mesurer les motivations au } \\
\text { travail. Par conséquent, elle } \\
\text { fait uniquement référence au } \\
\text { degré d'autodétermination et } \\
\text { pas au degré } \\
\text { d'altruisme/égoïsme. }\end{array}$ \\
\hline Décision & $\begin{array}{l}\text { Non retenue : au regard des } \\
\text { regroupements de dimensions } \\
\text { observés dans la littérature, il } \\
\text { semble plus judicieux } \\
\text { d'utiliser une échelle fondée } \\
\text { sur la théorie de } \\
\text { l'autodétermination }\end{array}$ & $\begin{array}{l}\text { Retenue } \\
\text { simplification des } \\
\text { degrés } \\
\text { d'autodétermination } \\
\text { (autodéterminées/non } \\
\text { autodéterminées) }\end{array}$ & $\begin{array}{l}\text { Non retenue au regard } \\
\text { des limites évoquées ci- } \\
\text { dessus }\end{array}$ & $\begin{array}{l}\text { Non retenue car moins } \\
\text { complète que l'échelle de } \\
\text { Chantal et Vallerand (2000), } \\
\text { mais conforte dans la théorie à } \\
\text { mobiliser. }\end{array}$ \\
\hline
\end{tabular}

En synthèse, il existe plusieurs échelles de mesure des motivations au bénévolat. La VFI (Clary et al., 1996) apparaît la plus populaire dans la littérature. Cependant, elle néglige le critère de l'origine de la motivation (choix ou contrainte). Aussi, certains auteurs qui l'utilisent opèrent des regroupements d'items en se fondant sur des critères proches de ceux de l'EMAB, notamment le degré d'altruisme/égoïsme (Agostinho et Paço, 2012) ou le caractère intrinsèque ou extrinsèque des motivations (Finkelstein, 2009), critère résultant de la théorie de l'autodétermination. Cette théorie semble d'ailleurs connaître un récent regain d'intérêt dans les recherches sur le bénévolat (Bidee et al., 2013 ; Haivas, Hofmans et Pepermans, 2012). Cependant, ces dernières mobilisent un instrument de mesure non spécifique au bénévolat, la Motivation At Work Scale - Revised (MAWS-R ; Gagné et al., 2015), Comme son nom l'indique, elle a été conçue, à l'origine, pour mesurer la motivation au travail. A notre connaissance, seule l'EMAB (Chantal et Vallerand, 2000) combine les critères d'altruisme/égoïsme et d'autodétermination, d'où le choix de cette échelle dans notre recherche. 
Tableau 1 - Caractéristiques distinctives des pressions temporelles

\begin{tabular}{|c|c|c|c|}
\hline $\begin{array}{l}\text { Pression } \\
\text { temporelle }\end{array}$ & Situationnelle (PTS) & Chronique (PTC) & Ultime (PTU) \\
\hline Echéance & Oui : Interne ou externe & $\underline{\text { Non }}$ & 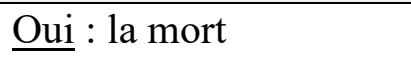 \\
\hline Durée & $\begin{array}{l}\text { Courte : Jusqu'à la date } \\
\text { de l'échéance }\end{array}$ & $\begin{array}{l}\text { Longue : En perpétuel } \\
\text { recommencement } \\
\text { chaque jour }\end{array}$ & $\begin{array}{l}\text { Inconnue à tendance } \\
\text { longue : Jusqu'au } \\
\text { décès de l'individu }\end{array}$ \\
\hline $\begin{array}{l}\text { Individus } \\
\text { concernés }\end{array}$ & $\begin{array}{l}\text { Personnes soumises à } \\
\text { une échéance (interne } \\
\text { ou externe) pour une } \\
\text { tâche précise } \\
\text { (un travail, un achat, un } \\
\text { projet à réaliser) }\end{array}$ & $\begin{array}{l}\text { Personnes constamment } \\
\text { dans l'urgence. } \\
\text { Tend à se généraliser } \\
\text { dans la population } \\
\text { occidentale }\end{array}$ & $\begin{array}{l}\text { Personnes ayant } \\
\text { conscience que } \\
\text { l'échéance ultime se } \\
\text { rapproche, } \\
\text { généralement suite à un } \\
\text { événement tel que la } \\
\text { retraite, provoquant une } \\
\text { prise de conscience du } \\
\text { vieillissement }\end{array}$ \\
\hline $\begin{array}{l}\text { Temps } \\
\text { concerné }\end{array}$ & $\begin{array}{l}\text { Quantité de temps } \\
\text { restant avant une } \\
\text { échéance }\end{array}$ & $\begin{array}{l}\text { Quantité de temps au } \\
\text { quotidien } \\
\text { (sans échéance) }\end{array}$ & $\begin{array}{l}\text { Quantité de temps } \\
\text { restant avant de mourir }\end{array}$ \\
\hline $\begin{array}{l}\text { Type de } \\
\text { réaction } \\
\text { affective }^{2}\end{array}$ & $\begin{array}{l}\text { Emotion, voire } \\
\text { appréciation }\end{array}$ & Sentiment & Sentiment \\
\hline
\end{tabular}

\footnotetext{
${ }^{2}$ Les réactions affectives peuvent être positives ou négatives quel que soit le type de pression temporelle. L'expression « réactions affectives » désigne une catégorie générique, englobant des émotions, des sentiments et des humeurs (Derbaix et Poncin, 2005). Lichtlé et Plichon (2014) rappellent certaines caractéristiques des émotions : elles sont généralement provoquées par un stimulus externe à l'individu (ex : l'atmosphère d'un point de vente) et de courte durée. Ainsi, le qualificatif d' "émotion » semble pertinent pour désigner le volet affectif de la pression temporelle situationnelle, de courte durée et généralement liée à un événement extérieur à l'individu. En revanche, un sentiment s'inscrirait davantage dans la durée (Derbaix et Pham, 1989) et s'associerait plus fortement à des mécanismes cognitifs. En raison du caractère durable de la PTU, la réaction affective associée ne peut donc être une émotion. Le terme «sentiment» semble alors adapté, en raison de son caractère plus intellectualisé et de sa durabilité.
} 
Tableau 2 - Résultats des analyses factorielles exploratoires et confirmatoires.

\begin{tabular}{|c|c|c|c|}
\hline \multicolumn{4}{|c|}{ Synthèse ACP, échantillon $\mathrm{n}^{\circ} 2$ (180 individus) } \\
\hline & $\begin{array}{c}\text { Axe } 1 \text { (dim. affective } \\
\text { négative) }\end{array}$ & $\begin{array}{c}\text { Axe } 2 \text { (dim. } \\
\text { cognitive) }\end{array}$ & $\begin{array}{l}\text { Axe } 3 \text { (dim. } \\
\text { affective positive) }\end{array}$ \\
\hline Valeur propre & 4,1 & 1,6 & 1,2 \\
\hline \% variance expliquée & $37 \%$ & $15 \%$ & $11,3 \%$ \\
\hline$\alpha$ de Cronbach & 0.772 & 0.784 & 0.771 \\
\hline Nombre d'items & 4 & 4 & 3 \\
\hline \multicolumn{4}{|c|}{ Synthèse AFC, échantillon $n^{\circ} 3$ (333 individus) } \\
\hline Modèle & Satorra-Bentler $\chi^{2} /$ dll & CFI*3 & RMSEA \\
\hline $1^{\mathrm{er}}$ ordre & $64,62 / 33=1.96$ & 0.973 & $0.054[0.034-0.073]$ \\
\hline $2^{\text {nd }}$ ordre & $73.60 / 40=1.84$ & 0.971 & $0.050[0.032-0.068]$ \\
\hline Alternatif unidimensionnel & $354.73 / 34=10,43$ & 0.722 & $0.169[0.153-0.184]$ \\
\hline Alternatif bidimensionnel & $185.63 / 34=5.46$ & 0.869 & $0.116[0.100-0.132]$ \\
\hline $\begin{array}{l}\text { Valeur conseillée } \\
\text { (Roussel et al., 2002) }\end{array}$ & $\begin{array}{l}\text { Le plus faible ; } \\
\text { entre } 1 \text { et } 2 \text { ou } 3 \text {; voire } 5\end{array}$ & $>0,90$ & $\begin{array}{l}<\text { à } 0,08 \text {, si possible } \\
<0,05\end{array}$ \\
\hline
\end{tabular}

3 Les statistiques robustes avec estimation du maximum de vraisemblance (ML) produisent des indices d'ajustement corrigés en fonction de l'intensité de la déviation de la multinormalité quand le coefficient de Mardia G2P Normalized estimate est supérieur à 3 (ici 5.5 et 8). 
Tableau 3- Fiabilité et validité de l'échelle PTU

\begin{tabular}{|c|c|c|c|c|c|c|}
\hline & \multicolumn{4}{|c|}{ Fiabilité } & \multicolumn{2}{|c|}{$\begin{array}{c}\text { Validité } \\
\text { convergente }\end{array}$} \\
\hline Dimension & \multicolumn{2}{|c|}{$\alpha$} & \multicolumn{2}{|c|}{$\rho$} & Test $\mathrm{z}$ & $\rho v c$ \\
\hline Cognitive & \multicolumn{2}{|c|}{0,83} & \multicolumn{2}{|c|}{0,83} & $>11$ & 0,56 \\
\hline Affective positive & \multicolumn{2}{|c|}{0,81} & \multicolumn{2}{|c|}{0,81} & $>13$ & 0,58 \\
\hline Affective négative & \multicolumn{2}{|c|}{0,76} & \multicolumn{2}{|c|}{0,76} & $>9$ & 0,46 \\
\hline Valeur conseillée (Hair et al., 2010) & \multicolumn{2}{|c|}{$>0.7$} & \multicolumn{2}{|c|}{$>0.7$} & $>1.96$ & $>0.5$ \\
\hline \multicolumn{7}{|c|}{ Validité discriminante } \\
\hline & $\begin{array}{l}\text { PTC (dim } \\
\text { cognitive) }\end{array}$ & $\begin{array}{c}\text { PTU } \\
\text { cognitive }\end{array}$ & $\begin{array}{c}\text { PTU } \\
\text { affective } \\
\text { positive }\end{array}$ & $\begin{array}{c}\text { PTU } \\
\text { affective } \\
\text { négative }\end{array}$ & $\begin{array}{c}\text { Anxiété } \\
\text { face à } \\
\text { la mort }\end{array}$ & $\begin{array}{c}\text { PTU 2 } 2^{\text {nd }} \\
\text { ordre }\end{array}$ \\
\hline PTC (dim cognitive) & 0,70 & & & & & \\
\hline PTU cognitive & 0,28 & 0,75 & & & & \\
\hline PTU affective positive & 0,23 & 0,40 & 0,76 & & & \\
\hline PTU affective négative & 0,45 & 0,41 & 0,52 & 0,68 & & \\
\hline Anxiété face à la mort & 0,24 & 0,33 & 0,38 & 0,59 & 0,80 & \\
\hline PTU $2^{\text {nd }}$ ordre & 0,49 & & & & 0,65 & 0,68 \\
\hline
\end{tabular}


Figure 1 : Influence de la PTU sur les motivations au bénévolat des retraités

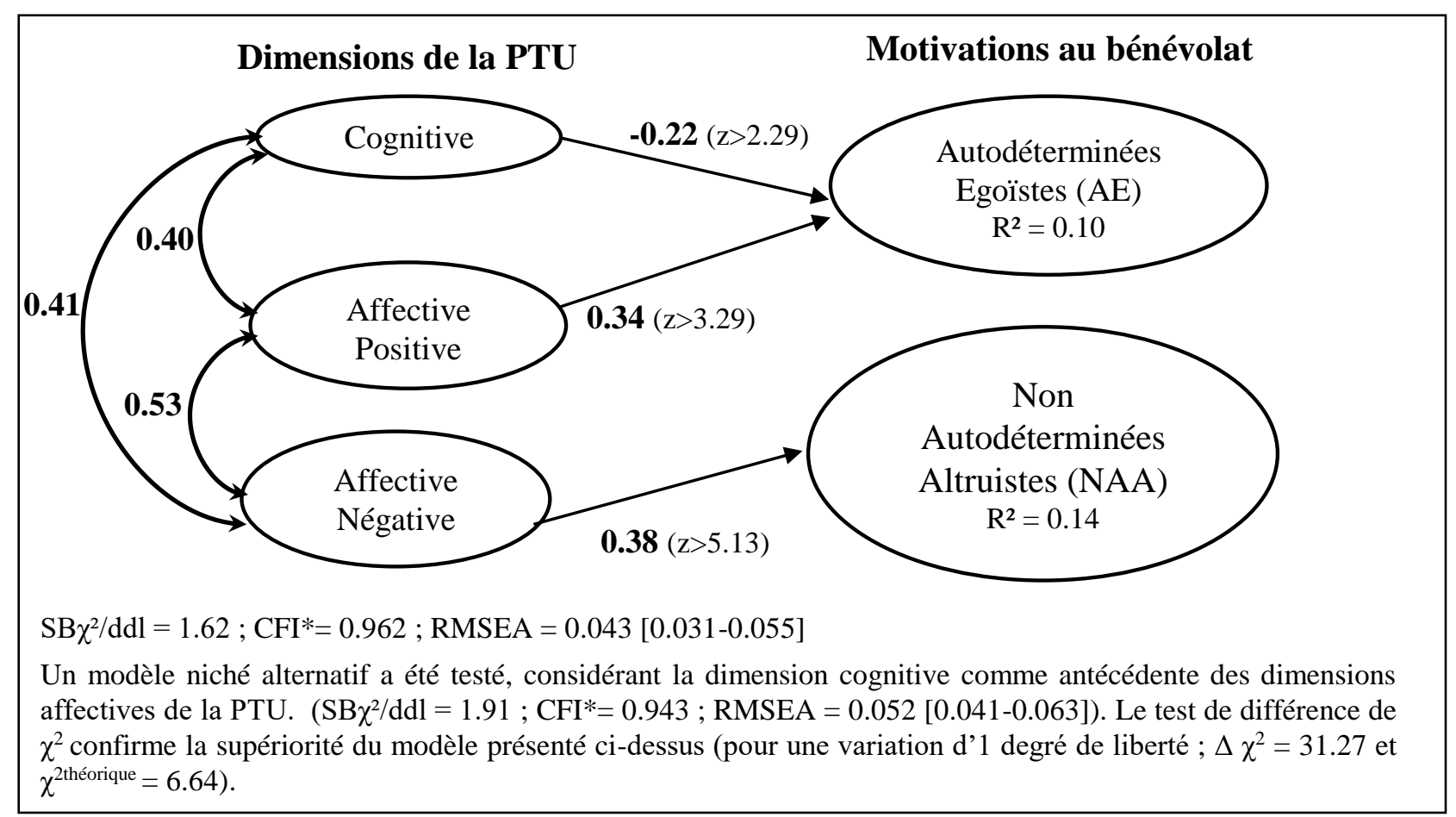




\section{BIBLIOGRAPHIE}

Adjaye JK (2002) Modes of knowing: intellectual and social dimensions of time in Africa. Kronoscope 2(2): 199-224.

Agostinho D et Paço A (2012) Analysis of the motivations, generativity and demographics of the food bank volunteer. International Journal of Nonprofit and Voluntary Sector Marketing 17(3): 249-261.

Allard-Poesi F (2003) Coder les données. In: Giordano Y (ed) Conduire un projet de recherche : une perspective qualitative. Colombelles: EMS Management \& société, pp. 245-290.

Attias-Donfut C (2006) Temps social, temps individuel et vieillissement. In: Attias-Donfut C, Cyrulnik B, Klein E et Misrahi R (eds) Penser le temps pour lire la vieillesse. Paris: Presses Universitaires de France.

Balbo G (1989) D’un vieillissement outre âge. In: Bianchi H (ed) La question du vieillissement : perspectives psychanalytiques. Paris: Dunod, pp.74-106.

Bazeley P (2007) Going further. In: Bazeley P (ed) Qualitative data analysis with NVivo. London: Sage Publications, pp.177-209.

Bergadaà M (1988) Le temps et le comportement de l'individu (Part 1). Recherche et Applications en Marketing 3(4): 57-72.

Bergadaà M (1989) Le temps et le comportement de l'individu (Part 2). Recherche et Applications en Marketing 4(1): 37-55.

Bergadaà M (1990) The role of time in the action of the consumer. Journal of Consumer Research 17(3): 289-302.

Bergadaà M et Urien B (2008) La personne âgée : son attitude face à l'idée de mort, le cadre temporel de son action et son sens de l'objet. In: Actes de la conférence Rupture, finitude, mort et Management, Brest, France, 25-26 juin 2008.

Bianchi H (1987) Le Moi et le temps : psychanalyse du temps et du vieillissement. Paris: Bordas.

Bianchi H (1989) Vieillir, ou les destins de l'attachement. In: Bianchi H (ed) La question du vieillissement : perspectives psychanalytiques. Paris: Dunod, pp.33-63.

Bidee J, Vantilborgh T, Pepermans R, Huybrechts G, Willems J, Jegers M et Hofmans J (2013) Autonomous motivation stimulates volunteers' work effort: A self-determination 
theory approach to volunteerism. VOLUNTAS: International Journal of Voluntary and Nonprofit Organizations 24(1): 32-47.

Boulbry G, Jacob C et Guéguen N (2012) Effets des formats publicitaires classiques de l'internet sur les réactions cognitives, affectives et conatives de deux segments d'âges opposés. In: Actes du $28^{\text {ème }}$ congrès de l'Association Française du marketing, Brest, France, 9-11 mai 2012.

Bourcier-Béquaert B et De Barnier V (2011) Les émotions chez les personnes âgées : entre pression temporelle et effet d'expérience. In: Actes des $10^{\text {èmes }}$ Journées Normandes de la Recherche sur la Consommation, Rouen, France, 18 mars 2011.

Bourcier-Béquaert B et De Barnier V (2012) Le sens donné par les personnes âgées à leurs consommations. In: Guiot D et Urien B (ed) Comprendre le consommateur âgé : Nouveaux enjeux et perspectives. Bruxelles: De Boeck, pp.107-128.

Caradec V (2008) Les «personnes âgées », un groupe d'âge hétérogène. In: Caradec V (ed) Sociologie de la vieillesse et du vieillissement. Paris: A. Colin, pp.42-86.

Carstensen LL (1991) Selectivity theory: social activity in life-span context. In: Schaie KW et Lawton MP (ed) Annual review of gerontology and geriatrics 11, pp.195-217.

Carstensen LL (1992) Social and emotional patterns in adulthood: support for socioemotional selectivity theory. Psychology and Aging 7(3): 331-338.

Carstensen LL et Lang FR (1996) Future time perspective scale. Unpublished manuscript. Stanford University.

Centre d'études et de recherches sur la philantropie - Cerphi (2010) Les associations et les seniors bénévoles : nouvelles relations et nouvelles pratiques. Disponible sur : http://www.cerphi.org/etudes-et-recherches-disponibles/le-benevolat-les-benevoles/ (accédé le 08 janvier 2016).

Centre d'études et de recherches sur la philantropie - (2012), L'engagement bénévole des seniors - de 50 à 65 ans en France. Disponible sur: http://www.aveengagements.fr/IMG/pdf/Sondage_Engagement_des_seniors-2.pdf (accédé le 08 janvier 2016).

Chantal Y et Vallerand RJ (2000) Construction et validation de l'échelle de motivation envers l'action bénévole (EMAB). Society and Leisure 23: 477-508.

Chevalier C, Le Serre D et Lichtlé MC (2013) La recherche d'information des seniors : caractéristiques et typologie. In: Actes du $29^{\text {ème }}$ congrès de l'Association Française du Marketing, La Rochelle, France, 14-15 mai 2013. 
Churchill GA (1979) A paradigm for developing better measures of marketing constructs. Journal of marketing research 16(1): 64-73.

Clary EG, Snyder M et Stukas AA (1996) Volunteers' motivations: findings from a national survey. Nonprofit and Voluntary Sector Quarterly 25(4): 485-505.

Clément S (2007) Le discours sur la mort à l'âge de la vieillesse. Retraite et Société, 3(52): 63-81.

Dávila MC et Díaz-Morales JF (2009) Age and motives for volunteering: Further evidence. Europe's Journal of Psychology (2): 82-95.

De Hennezel M (2010) Vieillir. In: De Hennezel M et Vergely B (ed), Une vie pour se mettre au monde. Paris: Carnets nord, pp.11-62.

Delacroix E et Jourdan P (2007) La tendance à regretter du consommateur : validation d'une échelle de mesure. Recherche et Applications en Marketing 22(1): 25-44.

Derbaix C et Pham MT (1989) Pour un développement des mesures de l'affectif en marketing : synthèse des prérequis. Recherche et Applications en Marketing 4(4): 71-87.

Derbaix C et Poncin I (2005) La mesure des réactions affectives en marketing, évaluation des principaux outils. Recherche et Applications en Marketing. 20(2): 55-75.

Erikson E (1959) Identity and the life cycle: Selected papers. Psychological Issues. 1: 50-100. Erlinghagen M et Hank K (2005) Participation of older europeans in volunteer work. Papier de recherche, Mannheim research institute for the economics of aging (MEA), 71: pp.1-27. Disponible sur : http://econpapers.repec.org/paper/meameawpa/05071.htm (accédé le 08 janvier 2016).

Erlinghagen M (2010) Volunteering after retirement. Evidence from German panel data. European Societies, 12(5): 603-625.

Finkelstein MA (2009) Intrinsic vs. extrinsic motivational orientations and the volunteer process. Personality and Individual Differences. 46(5-6): 653-658.

Fontaine R (2007) Psychologie du vieillissement. $2^{\mathrm{e}}$ édition. Paris: Dunod.

Fromage B (2007) Approche du vieillissement à travers l'expérience subjective. L'information psychiatrique. 83(3): 229-233.

Gagey J (1989) Raisonner psychanalytiquement le vieillir? In Bianchi H (ed) La question du vieillissement : perspectives psychanalytiques. Paris: Dunod, pp.7-32.

Gagné M, Forest J, Vansteenkiste M, Crevier-Braud L, Van den Broeck A, Aspeli A, Bellerose J, Bénabou C, Chemolli E, Güntert ST, Halvari H, Johnson P, Indiyastuti DL, Molstad M, Naudin M, Ndao A, Roussel P, Westbye C et Wang Z (2015) The 
multidimensional work motivation scale: Validation evidence in seven languages and nine countries. European Journal of Work and Organizational Psychology. 24(2): 178-196.

Gourmelen A et Guillemot S (2012) Pourquoi donner son temps lorsque celui-ci est compté ? L'influence de la générativité sur les motivations des bénévoles retraités. In: Actes du $28^{\text {ème }}$ congrès de l'Association Française du Marketing, Brest, France, 9-11 mai 2012.

Guiot D (2006) Un cadre d'analyse du processus de vieillissement et de son influence sur le comportement d'achat du consommateur âgé. Recherche et Applications en Marketing 21(1): 57-79.

Hair JF, Black WC, Babin BJ et Anderson RE (2010) Multivariate data Analysis (7 ${ }^{\text {ème }}$ édition). Prentice Hall.

Haivas S, Hofmans J et Pepermans R (2012) Self-determination theory as a framework for exploring the impact of the organizational context on volunteer motivation: A study of romanian volunteers. Nonprofit and Voluntary Sector Quarterly 41(6): 1195-1214.

Haski-Leventhal D (2009) Elderly volunteering and well-being: a cross-European comparison based on SHARE data. VOLUNTAS: International Journal of Voluntary and Nonprofit Organizations 20(4): 388-404.

Huberman AM et Miles MB (1991) Analyse pendant le recueil des données. In: Huberma AM et Miles MB (ed) Analyse des données qualitatives - Recueil de nouvelles méthodes. Bruxelles: De Boeck, pp.88-138.

Huteau M (2006) Psychologie différentielle : cours et exercices. $3^{\mathrm{e}}$ édition. Paris: Dunod.

Ionescu S, Jacquet MM et Lhote C (2012) Les mécanismes de défense : théorie et clinique. 2e édition Paris: A. Colin.

Jolibert A et Jourdan P (2011a) Les techniques d'analyse qualitatives. In Jolibert A et Jourdan P (ed) Marketing research, méthodes de recherche et d'études en marketing. Paris: Dunod, pp.49-65.

Jolibert A et Jourdan P (2011b) La construction d'une échelle de mesure. In Jolibert A et Jourdan P (ed) Marketing research, méthodes de recherche et d'études en marketing. Paris: Dunod, pp.178-198.

Lalive d'Epinay C et Cavalli S (2007) Changements et tournants dans la seconde moitié de la vie. Gérontologie et Société. 2(121): 45-60.

Lallement $\mathrm{J}$ (2008) Les effets de la pression temporelle sur le traitement des informations et le comportement d'achat. Thèse de doctorat, Université de Tours, France. 
Lallement J (2010) L'impact de la pression temporelle sur le traitement des informations. Recherche et Applications en Marketing 25(4): 45-68.

Lallement J et Machat S (2011) «Au secours, tout va trop vite ! » Nécessité d'une conceptualisation marketing de la pression temporelle chronique. In: Actes du $27^{7_{m}}$ congrès de l'Association Française du Marketing, Bruxelles, Belgique, 19-20 mai 2011.

Lichtlé MC et Plichon V (2014) Les émotions ressenties dans un point de vente : Proposition d'une échelle de mesure. Recherche et Applications en Marketing 29(1): 3-26.

Machat S et Lallement J (2011) Vite fait, bien fait ? Proposition de mesure de la pression

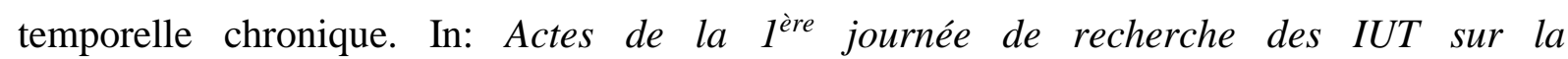
commercialisation, Saint-Denis, France, 3 mars 2011.

Malas Z (2009) Le rapport à l'avenir, déterminant des choix patrimoniaux des personnes âgées. Thèse de doctorat, Université de Paris Dauphine, Paris, France.

Malas Z et Guiot D (2008) L'étude du rapport à l'avenir en marketing chez les plus de 50 ans : différences conceptuelles et mesures alternatives. In: Actes du $24^{\text {ème }}$ congrès de l'Association Française du Marketing, Paris, France, 15-16 mai 2008.

Malas Z et Safraou I (2008) La mesure de l'horizon temporel par Carstensen et Lang : discussion, traduction et validation de l'échelle sur une population française de plus de 50 ans. In: Actes de la conférence Rupture, finitude, mort et Management, Brest, France, 25-26 juin 2008.

Marbot É et Peretti JM (2004) Les seniors dans l'entreprise. Paris: Village Mondial, Pearson Education France.

Marchand I, Quéniart A et Charpentier M (2010) Vieillesses d'aujourd'hui : les femmes aînées et leurs rapports aux temps. Enfances, Familles, Générations 13: 59-78.

McAdams DP, de St Aubin E et Logan RL (1993) Generativity among young, midlife, and older adults. Psychology and aging, 8: 221-230.

Miles MB et Huberman AM (2003) Donner un sens : élaboration et vérification des conclusions. In Miles MB et Huberman AM (ed) Analyse des données qualitatives. Bruxelles: De Boeck, pp.437-518.

Misrahi R (2006) Conversion et temps vécu : vieillesse, gérontologie, philosophie. In AttiasDonfut C, Cyrulnik B, Klein E et Misrahi R (ed) Penser le temps pour lire la vieillesse. Paris : Presses Universitaires de France.

Morrow-Howell N (2010) Volunteering in later life: research frontiers. The Journals of Gerontology, Series B, Psychological Sciences and Social Sciences 65(4): 461-469. 
Moschis GP et Nickell D (2012) Comprendre les motivations d'entrée dans un établissement d'hébergement médicalisé : le cas des Etats-Unis. In Guiot D et Urien B (ed) Comprendre le consommateur âgé : nouveaux enjeux et perspectives. Bruxelles: De Boeck, pp.179-194.

Mutchler JE, Burr JA et Caro FG (2003) From paid worker to volunteer: leaving the paid workforce and volunteering in later life. Social Forces 81(4): 1267-1293.

O’Neill G, Morrow-Howell N et Wilson SF (2011) Volunteering in later life: from disengagement to civic engagement. In: Settersten RA Jr et Angel JL (ed) Handbook of Sociology of Aging. Springer, pp.333-350.

Okun MA et Schultz A (2003) Age and motives for volunteering: testing hypotheses derived from socioemotional selectivity theory. Psychology and aging 18(2): 231-239.

Okun MA, Barr A et Herzog AR (1998) Motivation to volunteer by older adults: a test of competing measurement models. Psychology and Aging 13(4): 608-621.

Paillé P et Mucchielli A (2008) L'examen phénoménologique des données empiriques. In: Paillé P et Mucchielli A (ed) L'analyse qualitative en sciences humaines et sociales. Paris: Armand Colin, pp.85-98.

Point S et Voynnet-Fourboul C (2006) Le codage à visée théorique. Recherche et Applications en Marketing 21(4): 61-78.

Prouteau L et Wolff FC (2004) Donner son temps: les bénévoles dans la vie associative. Economie \& Statistique 372: 3-39.

Prouteau L et Wolff FC (2007) La participation associative et le bénévolat des seniors. Retraite et Société 1(50): 157-189.

Pyszczynski T, Greenberg J et Solomon S (1999) A dual-process model of defense against conscious and unconscious death-related thoughts: an extension of terror management theory. Psychological Review 106(4): 835-845.

Reed AE et Carstensen LL (2012) The theory behind the age-related positivity effect. Frontiers in psychology 3: 1-9.

Rizkalla AN (1989) Sense of time urgency and consumer well-being: testing alternative causal models. In: Advances in Consumer Research, 16, pp.180-188.

Rosa H (2010) Accélération et croissance : les forces motrices externes de l'accélération sociale. In Rosa H (ed) Accélération : une critique sociale du temps. Paris: La Découverte, pp.199-239.

Rossiter JR (2002) The C-OAR-SE procedure for scale development in marketing. International Journal of Research in Marketing 19(4): 305-335. 
Rossiter JR (2011) Marketing measurement revolution: the C-OAR-SE method and why it must replace psychometrics. European Journal of Marketing 45: 1589-1600.

Roussel P, Durrieu F, Campoy É et El Akremi A (2002) Méthodes d'équations structurelles : recherche et applications en gestion. Paris: Economica.

Schau HJ, Gilly MC et Wolfinbarger M (2009) Consumer identity renaissance: The resurgence of identity-inspired consumption in retirement. Journal of Consumer Research 36(2): 255-276.

Schwartz B, Ward A, Monterosso J, Lyubomirsky S, White K et Lehman DR (2002) Maximizing versus satisficing: happiness is a matter of choice. Journal of Personality and Social Psychology 83(5): 1178-1197.

Szmigin I et Carrigan M (2001) Time, consumption, and the older consumer: an interpretive study of the cognitively young. Psychology and Marketing 18(10): 1091-1116.

Szollos A (2009) Toward a psychology of chronic time pressure. Time \& Society 18(2-3): 332-350.

Talpin JM (2013) Psychologie clinique du vieillissement normal et pathologique. Paris: A. Colin.

Tang F, Morrow-Howell N et Hong S (2009) Inclusion of diverse older populations in volunteering. Nonprofit and Voluntary Sector Quarterly 38(5): 810-827.

Thomas LV (1989) Attitudes collectives envers les vieillards : un problème de civilisation. In H. Bianchi (ed) La question du vieillissement : perspectives psychanalytiques. Paris: Dunod, pp.125-160.

Tolstoï L (1986), Je m'en vais - lettre 602 à la comtesse S.A. Tolstaïa. In: Christian RF (ed) Lettres II, Gallimard, pp.385-386.

Urien B (2002) L'anxiété face à la mort : approfondissement conceptuel et étude de son influence sur la recherche de variété alimentaire. In: Actes du $18^{\text {ème }}$ congrès de l'Association Française du Marketing, Lille, France, 22-23 mai 2010.

Urien B (2003) L'anxiété face à la mort et son influence sur le comportement du consommateur. Recherche et Applications en Marketing 18(4): 23-41.

Urien B (2007) Selfless time, from life space to death anticipation. Time \& Society 16 (2-3): 367-386.

Usunier JC et Valette-Florence P (2007) The time styles scale. A review of developments and replications over 15 years. Time \& Society 16(2-3): 333-366. 
Van Hiel A, Mervielde I, et De Fruyt F (2006) Stagnation and Generativity: structure, validity, and differential relationships with adaptive and maladaptive personality. Journal of Personality 74(2): 543-574.

Van Ingen E et Dekker P (2011) Changes in the determinants of volunteering: participation and time investment between 1975 and 2005 in the Netherlands. Nonprofit and Voluntary Sector Quarterly 40(4): 682-702.

Vergely B (2010) Mûrir. In: De Hennezel M et Vergely B (ed) Une vie pour se mettre au monde. Paris: Carnets nord, pp.63-118.

Wang PZ (2004) Assessing motivations for sports volunteerism. In: Advances in Consumer Research, 31, pp.420-425.

Warburton J et Crosier T (2001) Are we too busy to volunteer? The relationship between time and volunteering using the 1997 ABS time use data. Australian Journal of Social Issues 36(4): 295-314.

Wiedmer JP (2010) Les seniors, des passeurs de vie. In Wiedmer JP (ed) Enfin senior!, Paris: Nouveaux débats publics, pp.189-209.

Zedlewski SR et Schaner SG (2006) Older adults engaged as volunteers. Perspectives On Productive Aging 5: 1-7.

Zeelenberg M (1999) The use of crying over spilled milk: a note on the rationality and functionality of regret. Philosophical Psychology 12(3): 325-340. 